\title{
Digital PCR quantification of DNA, RNA and extracellular microRNA of mouse oocytes
}

Joan Xiaohui Yang ${ }^{1,2}$, Xin Yuan Zhao ${ }^{3}$, Dexi $\mathrm{Bi}^{4}$, Qing Wei ${ }^{4}$, Citra Mattar ${ }^{5}$, Joy Yan Ling Pang $^{3}$, Yie Hou Lee $2,6,7, \ddagger$

${ }^{1}$ Division of Obstetrics \& Gynaecology, KK Women's and Children's Hospital, Singapore, Singapore

${ }^{2}$ Translational ‘Omics and Biomarkers Group, KK Research Centre, KK Women's and Children's Hospital, Singapore, Singapore

${ }^{3}$ Chemical Engineering \& Food Technology, Singapore Institute of Technology,

Singapore, Singapore

${ }^{4}$ Department of Pathology, Shanghai Tenth People's Hospital, Tongji University School of Medicine, Shanghai 200072, China

${ }^{5}$ Department of Obstetrics \& Gynaecology, Yong Loo Lin School of Medicine, National University of Singapore, Singapore, Singapore

${ }^{6}$ Obstetrics \& Gynaecology Academic Clinical Program, Duke-NUS Medical School, Singapore, Singapore

${ }^{7}$ Critical Analytics in Manufacturing Precision Medicine, Singapore-MIT Alliance for Research and Technology, Singapore

\section{${ }^{\ddagger}$ Address for correspondence and re-print requests:}

Yie Hou Lee. Ph.D. Phone: (+65) 6394 8122. Email: yiehou.lee@smart.mit.edu

Funding: SingHealth Foundation (SHF/FG560P/2014) and National Medical Research Council, Singapore (NMRC/BNIG/2033/2015).

Disclosure summary: All authors have nothing to disclose.

Short running title: Absolute DNA/RNA quantification by digitalPCR

Keywords: Oocyte; ceramide; digital PCR; extracellular microRNA; endometriosis, 


\section{ABSTRACT}

Despite numerous advances in in vitro fertilization (IVF) techniques since its first success in 1978, almost half of the patients treated remain childless. The multifactorial nature of IVF treatment means that success is dependent on variables, including the quality of oocytes. Therefore, new technologies are needed to objectively and quantitatively examine how each oocyte can be selected or optimized to achieve for the best possible outcomes for patients. Here, we report an optimized digital polymerase chain reaction (dPCR) for direct absolute quantification of nucleic acids within $3.5 \mathrm{~h}$ without the need for sample extraction or purification. Using individual oocytes, the developed method demonstrated absolute quantification with a linear dynamic range of $0.65-33$ copies $/ \mu \mathrm{L}$ $\left(r^{2}=0.999\right)$, high accuracy and excellent reproducibility of $<10 \%$ relative standard deviation. The method then identified the variable expression of Gapdh (0.72-16.95 copies/oocyte), Hprt1 (1.05-19.05 copies/oocyte) and ATPase 6, (5.55-32358.15 copies/oocyte) in ovaries even from the same mouse. Finally, dPCR was used to validate extracellular microRNAs from oocytes incubated with a toxic unsaturated very-long chained ceramide. This study therefore shows the feasibility of $\mathrm{dPCR}$ for the rapid and sensitive absolute quantification of DNA/RNA and extracellular miRNA for the study of oocytes.

(194 words) 


\section{INTRODUCTION}

The use of assisted reproductive technology (ART) has risen steadily, with more than 8 million babies born from in vitro fertilization (IVF) and intracytoplasmic sperm injection (ICSI) since 1978 [1]. With an average of 2.7 treatment cycles required before pregnancy [2] and ART pregnancy rates stabilizing at $36 \%$, improvements are been sought to improve ART outcomes. While ART offers the highest success rate in achieving pregnancy for infertility couples, the take-home baby rate remains disappointingly low [3]. The technology of IVF consists mainly of pick up, selection and insemination of oocytes, embryo culture and their transfer into recipient's womb. Before any further manipulation of oocytes their quality must be accurately evaluated as it has direct impact on the monospermic fertilization, early development, establishment and maintenance of pregnancy. The main challenge related to selecting high quality oocytes is that developmentally incompetent oocytes may exhibit the same morphologies as the good ones.

The oocyte is a unique and highly specialized cell that supports cellular homeostasis, metabolism, and cell cycle progression in the early embryo, as well as being necessary for creating, activating, and controlling the embryonic genome. During oogenesis, under the instructions of eight transcriptome factors, each oocyte undergoes homologous recombination of paternal and maternal genomes reciprocally exchanging DNA between homologous chromosomes to generate crossovers [4]. Hence, each germ cell has a unique genome that contributes to genetic diversity, resulting in the natural selection of the most competent oocyte to mature and ovulate during each estrous cycle $[5,6]$. Disruptions in oogenesis through genetic, environmental factors and gynecological disorders can compromise oocyte quality, leading to arrested development and reduced fertility that affect long-term health of the offspring [7]. A viable, high quality oocyte is therefore a prerequisite for successful fertilization and implantation. Oocyte maturation also requires extensive signalling between germ cells and their surrounding theca cells, mural granulosa cells, cumulus cells, all secreting biological factors within the ovarian follicular microenvironment.

Recent studies have revealed the presence of cell-secreted microvesicles and exosomes in follicular fluids, of which extracellular microRNAs (exmiRNAs) are the most abundant constituents [8-10]. miRNAs are a class of small non-coding endogenous RNAs of 19-22 nucleotides that act as post-transcriptional regulators of gene expression [11]. Mature miRNAs are loaded onto the Argonaute (Ago) protein to form the miRNA-induced silencing complex (RISC). miRNA directs RISC to its target mRNA through Watson-Crick base 
pairing, promoting mRNA destabilization, degradation and translational inhibition [12]. Dysregulated expression of miRNAs such as miR-206, miR-574, let-7e-5p, miR-29a-3p, miR126-3p, miR-136-5p, miR-192-5p, miR-203a-3p have frequently been related with oocyte development and aging [13-16]. Our expanded understanding of the molecular determinants of oocyte quality and how these determinants can be disrupted can reveal new insights into the role of oocyte functions in development and improve oocyte development. Therefore, the ability to conduct accurate and sensitive oocyte genetic analysis is critical for gaining insights to oocyte maturation, development, and aging, serving as a basis for understanding infertility disorders and improving fertility outcomes.

In recent years, digital PCR ( $\mathrm{dPCR}$ ) has gained popularity in quantifying genetic material [17-19]. The technique of partitioning allows for high signal-to-noise ratio, high sensitivity to quantify absolute target genes, and being calibration free, are major advantages of dPCR over that of quantitative PCR (qPCR) [20,21]. In addition, dPCR overcomes the shortcomings in qPCR, such as the need for the normalization of the target gene-ofinterest to endogenous reference or housekeeping genes, and the need for extraction, both of which result in inaccurate quantification and hampers inter-laboratory comparability of data [22,23] [24]. While direct DNA quantification from plasma or serum using dPCR was previously shown [21,25-27], one step direct detection and RNA quantification in oocytes has not yet been successfully shown. Single oocyte places a physical limitation on RNA isolation due to its minute cellular and genetic volume, as well as the risk of loss. In this study, we optimized a dPCR protocol that is able to provide an absolute quantification of messenger RNA (mRNA) from single oocytes without the need for sample extraction or purification, i.e. direct quantification. Further, we demonstrated the noninvasive application of dPCR in quantifying extracellular miRNAs in ceramide (Cer) murine oocytes exposed to ceramide (Cer) [28]. 


\section{METHODS}

\section{Chemicals}

Chemicals, reagents and instruments were from Thermo Scientific unless otherwise stated.

\section{Murine Oocyte and Lung Harvesting}

BALB/c female mice were maintained in a temperature and light controlled, pathogen-free space $\left(24^{\circ} \mathrm{C}, 14 \mathrm{~h}\right.$ light/10 h dark cycles) with ad libitum food and water. Balb/C female mice (4-8 weeks old) were sacrificed by cervical dislocation and the ovaries were harvested under NUS IACUC protocol R15-1160. The ovaries were collected in oocyte manipulating media [alpha Minimum Essential Medium alpha ( $\alpha M E M)+$ GlutaMax media (Gibco, Life Technologies, Carlsbad, CA) supplemented with 25 mM HEPES (Gibco)] and punctured repeatedly with a 30-guage needle to release the cumulus-oocyte complexes (COCs). The COCs were the collected using stripper tips (Origio, CooperSurgical, Charlottesville, VA) with an internal diameter (ID) of $100 \mu \mathrm{m}$ attached to the STRIPPER $\circledast$ micropipetter (Origio), and place in fresh manipulating media. The surrounding cumulus cells were removed mechanically using ID $75 \mu \mathrm{m}$ stripper tips. Denuded oocytes were transferred to fresh media and healthy denuded germinal vesicle (GV) oocytes were collected based on their morphology: (i) presence of GV, (ii) oocyte size and shape, and (iii) perivitelline space (PVS). Batches of denuded GV oocytes were rinsed twice in DEPC water $+1 \%$ SUPERase in RNase Inhibitor $\left(\right.$ Ambion $\left.^{\mathrm{TM}}\right)$. Several types of buffers were evaluated as part of the dPCR optimization process, namely, RNAlater, DEPC (Invitrogen), DPBS (Lonza), DPBS with RNase inhibitor (SUPERase In RNase Inhibitor), and two types of oocyte culture media: $\alpha M E M$ and in vitro maturation (IVM) media [SAGE oocyte maturation media (CooperSurgical, Trumbull, CT)]. $2 \mu \mathrm{L}$ of buffer was pipetted onto a culture dish and a single denuded GV oocyte was transferred to the droplet. The oocyte and droplet of buffer was then transferred to a PCR tube (total volume of $6 \mu \mathrm{L}$ ) and stored at $-80^{\circ} \mathrm{C}$ until further analysis. As part of the method optimization process, murine lungs were harvested. Lung tissues were washed in PBS and separated into different tubes with the respective buffers. The tissues were then homogenized using Qiagen TissueLyser II at $1 \mathrm{~min}, 30 \mathrm{~Hz}, 3$ cycles with TissueLyser $5 \mathrm{~mm}$ beads. Homogenized tissues were stored at $-80^{\circ} \mathrm{C}$ before analysis (within one week).

\section{Oocyte Culture Supernatant}

Oocyte culture supernatant was collected as previously described [28]. Briefly, groups of 25 denuded GV oocytes were cultured in $30 \mu \mathrm{L}$ of IVM media supplemented with 25 $\mathrm{mIU} / \mathrm{mL}$ pregnant mare's serum gonadotropin (PMSG; Sigma, St. Louis, MO) and 25 
$\mathrm{mIU} / \mathrm{mL}$ human chorionic gonadotropin [hCG (Pregnyl); MSD, Oss, The Netherlands]. The culture drops were layered with mineral oil and matured for $14 \mathrm{~h}$ at $37^{\circ} \mathrm{C}$ in a humidified atmosphere of $5 \% \mathrm{CO}_{2} .60 \mathrm{ng} / \mathrm{mL} \mathrm{C} 24: 1$-Cer was spiked into the IVM media of the Cer group. After $14 \mathrm{~h}$, the culture supernatant was collected and stored at $-80{ }^{\circ} \mathrm{C}$.

\section{cDNA Conversion}

To convert mouse lung mRNA to complementary DNA (cDNA), a mastermix which comprised of Moloney Murine Leukemia Virus Reverse Transcriptase (M-MLV RT), MMLV RT $5 \times$ Reaction Buffer, RNAsin Ribonuclease inhibitor, random primers, dNTP mix and RNAse-free water was prepared (Table S1). Reverse transcription (RT) was ran at $37^{\circ} \mathrm{C}$ for $60 \mathrm{~min}$. cDNA concentrations were analyzed using a NanoDrop 2000 spectrophotometer.

\section{Quantitative Real-Time Polymerase Chain Reaction}

Primers for mRNA quantification were designed to bind to non-adenlyated mRNA sequences. Primer sequences are shown in Table S2. Each reaction mix consists of 150 $\mathrm{nM}$ forward and reverse primers and 1× SYBR Green PCR Master Mix (Applied Biosystems $^{\mathrm{TM}}$ ), $3 \mu \mathrm{L}$ cDNA sample and PCR grade water topped up to $15 \mu \mathrm{L}$ (Table S1). The reaction mix were then ran at the following cycling conditions: (i) polymerase activation $-95^{\circ} \mathrm{C}$ for $5 \mathrm{~min}$, (ii) denaturing / annealing \& extension $-95^{\circ} \mathrm{C}$ for $50 \mathrm{~s}$ and $58^{\circ} \mathrm{C}$ for $90 \mathrm{~s}$ for 40 cycles. Amplification of the cDNA were analyzed using the Applied Biosystems 7500 Fast Real-Time PCR system. The threshold cycle $\left(\mathrm{C}_{\mathrm{T}}\right)$ was used to determine the relative abundance of the targets.

\section{Digital Polymerase Chain Reaction}

Commercial chips for sample partitioning and matched fluorescence reader for endpoint detection were used (Clarity dPCR system) [26]. For dPCR of mouse lung cDNA, $150 \mathrm{nM}$ of forward and reverse primers and 1× SYBR Green PCR Master Mix, 1× Clarity JN solution, $3 \mu \mathrm{L}$ DNA sample and PCR grade water topped up to $15 \mu \mathrm{L}$. The samples were then delivered onto the Clarity high-density chips using the Clarity auto loader, where it was sub-divided into 10,000 partitions. The partitions were then sealed with the Clarity Sealing Enhancer and $245 \mu \mathrm{L}$ of Clarity Sealing Fluid was added to each tube. The tube strips were transferred to a thermal cycler for PCR amplification using the following parameters: (i) polymerase activation: $95^{\circ} \mathrm{C}$ for $5 \mathrm{~min}$, (ii) denaturing / annealing \& extension: $95^{\circ} \mathrm{C}$ for $50 \mathrm{~s}$ and $58^{\circ} \mathrm{C}$ for $90 \mathrm{~s}$ for 40 cycles, (iii) final hold: $70^{\circ} \mathrm{C}$ for $5 \mathrm{~min}$. The ramp rate is kept at $1^{\circ} \mathrm{C} / \mathrm{s}$ throughout the cycling. The tube strips were allowed to cool to 
room temperature and transferred to the Clarity Reader which detects fluorescent signals from each partition concurrently. Imaged fluorescent signals are shown in Figure 1A, wherein positive and negative partitions are displayed as green and blue dots, respectively. In partitions that did not receive any reaction mixture are displayed as black backgrounds. The data were analysed with the Clarity software (version 2.1). Poisson statistics is used to determine the gene copy number per microlitre of sample based on positive signals. Data are expressed as DNA copy number per microlitre of sample or per sample itself. No template controls (NTC) were used as negative controls. From loading of samples to completion of data acquisition, the entire dPCR assay took approximately 3.5 hours.

\section{Digital PCR - One-Step mRNA Reverse Transcription}

This protocol utilizes direct detection of the transcripts, eliminating the need for the purification of mRNA (Figure 1B). Assessment of compatible buffers was conducted using $G A P D H$ as the model gene given its ubiquitous expression for the evaluation of the buffers. Firstly, $1 \mathrm{ng} / \mu \mathrm{L}$ of total DNA from mouse lung tissue was split and suspended in six different buffers/solutions, namely: RNAlater, DEPC, DPBS, DPBS with RNAse inhibitor and oocyte culture media, alphaMEM and IVM. The most optimal buffer, as evaluated based on readout of Gapdh, then went into a second round of buffer compatibility evaluation using mouse oocytes. DEPC was determined as the most compatible buffer.

Single oocytes in PCR tubes were thawed and incubated with $0.2 \mathrm{mg} / \mathrm{mL}$ of DNase I (Roche) at $37^{\circ} \mathrm{C}$ for $30 \mathrm{~min}$ on a shaker. The reaction mastermix were then added to the oocyte samples directly. Each mastermix consisted of $150 \mathrm{nM}$ forward and reverse primers, 1× QuantiNova RT Mix (Qiagen), 1× QuantiNova SYBR Green RT-PCR Master mix, 1× Clarity $^{\mathrm{TM}} \mathrm{JN}$ solution and PCR grade water topped up to $15 \mu \mathrm{L}$. The reaction mix were loaded onto the chips and sealed before transferring to a thermal cycler. Prior to thermal cycling (as detailed in above for DNA dPCR), a RT cycle of $50^{\circ} \mathrm{C}$ for $10 \mathrm{~min}$ was performed. Data are expressed as mRNA copy number per oocyte.

\section{Digital PCR - MicroRNA Reverse Transcription}

miRCURY LNA miRNA PCR Kit (Qiagen) was used to carry out RT-dPCR on the oocyte supernatant miRNA. To run RT, the supernatant was mixed with $5 \times \mathrm{RT}$ Reaction Buffer and 10× RT Enzyme Mix and transferred to a thermal cycler (Bio-Rad). Cycling conditions were as follows: (i) RT: $42^{\circ} \mathrm{C}$ for $60 \mathrm{~min}$, (ii) inactivation: $95^{\circ} \mathrm{C}$ for $5 \mathrm{~min}$. Following $\mathrm{RT}$ in PCR tubes, the miRNA cDNA samples were then added to the $\mathrm{APCR}$ reaction mix), loaded onto the Clarity chips, sealed and amplified as detailed above (in Digital Polymerase Chain Reaction sub-section), with an annealing temperature of $56^{\circ} \mathrm{C}$. miRCURY LNA PCR 
primers (Qiagen) were used for optimal PCR results. Synthetic miRNA sequences (Table S1) were designed to optimize the detection of miRNAs in the Clarity platform.

\section{Profiling of extracellular miRNome}

Total RNA was extracted from $20 \mu \mathrm{L}$ of pooled oocyte culture media using the miRNeasy Serum/Plasma kit (Qiagen) in a final elution volume of $10 \mu \mathrm{L}$. Subsequently, the extracted RNA was processed using the miScript Single Cell qPCR kit (Qiagen) according to the manufacturer's instructions with the following modifications. A total of $5.5 \mu \mathrm{L}$ extracted RNA was used as starting material for the 3 ' ligation reaction. The subsequent 5 ' ligation, universal cDNA synthesis, clean-up and universal preamplification steps were performed as per protocol. Sample quality was checked using five control miScript Primer Assays to monitor the 3' ligation, 5' ligation, reverse-transcription, preamplification and real-time PCR performance. All samples passed quality control.

The profiling of the 940 most abundantly expressed and best characterized miRNA sequences in the mouse miRNA genome (miRNome) as annotated in miRBase Release 16 (www.mirbase.org) was then performed using miScript Mouse miRNome PCR array (V16.0, 96-well/Rotor-Disc 100; Qiagen) in accordance with the manufacturer's instructions. Data analysis was performed using the online miScript miRNA PCR Array Data Analysis Tool. Briefly, from $20 \mu \mathrm{L}$ pooled mouse oocyte culture media from untreated $(N=3)$, or $C_{24: 1}$-Cer-treated individual mice $(N=3)$ [28], miRNA was extracted and converted to cDNA as described above. Next, the cDNA was diluted 1:10 in RNAse/DNAse free water and used as a template for miRNA PCR array amplification. Before that, the PCR array plate was sealed and centrifuged at $1,000 \times \mathrm{g}$ for $1 \mathrm{~min}$ at RT to remove air bubbles that may interfere with the PCR amplification process. Then, PCR reactions were performed using a thermo cycler (Bio-Rad, Hercules, CA, USA). The reaction mixtures were incubated at $95^{\circ} \mathrm{C}$ for $10 \mathrm{~min}$ to activate the HotStart DNA Taq polymerase, followed by 40 cycles of $95^{\circ} \mathrm{C}$ for $15 \mathrm{~s}$ and $60^{\circ} \mathrm{C}$ for $60 \mathrm{~s}$, monitored by melt curve analysis. $C_{t}$ values $>35$ were excluded from the analyses.

\section{Oocyte mRNA-sequencing}

Using mRNA from the same oocytes from untreated $(N=3)$, or $\mathrm{C}_{24: 1}$-Cer-treated individual mice $(N=3)$ [28] as with the miRNA profiling, library preparation was carried out according to manufacturer's instructions for QIAseq FX single-cell RNA Kit (Qiagen) using to $7 \mu \mathrm{L}$ of cell material as starting material. Libraries were quantified using PerfeCTa NGS Quantification Kit for Illumina (Quanta Biosciences) and pooled into a single library for 
paired end sequencing on one lane of HiSeq4000 using $151 \mathrm{bp}$ read length. Data analysis was then performed using the STAR-RSEM pipeline of GIS' NGS Pipeline Framework version $2017-10.0$

\section{Bioinformatics Analysis}

Prior to bioinformatics analysis, differently expressed mRNAs and miRNAs were identified with the limma package (version 3.10.3) in $\mathrm{R}$ (version 3.4.1) [29]. Spearman correlation was performed to identify negatively associated miRNA-mRNA pairs with the miRNA and mRNA expression profiling datasets of all samples as previously described [30] in $\mathrm{R}$ and those with $p<0.01$ were subsequently subject to miRNA target analysis with miRWalk2.0 [31], which integrated twelve existing miRNA-target prediction databases. The miRNAs with over ten target genes appearing in $\geq 6$ databases from the miRNA-mRNA pairs were selected and the target genes of each miRNA were subject to Gene Ontology enrichment analysis [32] and KEGG pathway analysis [33]. Gene functions was also cross-referenced with UniProt (https://www.uniprot.org/), when needed. Cytoscape (version 3.7.0) [34] was used to visualise miRNA-mRNA networks.

\section{Statistical Analysis}

Fold change was calculated as the average ratio of normalized molecular tag counts (or relative miRNA expression) between the untreated group and $\mathrm{C}_{24: 1}$-Cer-treated group. Numbers greater than 1 indicate upregulated or increased miRNA expression, numbers between 0 and 1 indicate downregulated or decreased miRNA expression, and a fold change of 1 indicates no change. $P$-values were calculated using a Student's t-test (twotail distribution and equal or unequal variances between the two samples) on the average delta $C_{t}$ values in each Test Group compared to the Control Group. 


\section{RESULTS}

\section{Direct digital DNA and RNA quantification optimization}

The direct DNA and RNA dPCR quantification method in single oocyte is shown in Figure 1. The development and optimization of the direct $\mathrm{APCR}$ analysis on the chip-based dPCR system consist of three main steps: (i) buffer selection, (ii) determination of quantification accuracy, and (iii) determination of quantification parameters. Assessment of the compatibility of various buffers was first determined using mouse lung tissues, then mouse oocytes. As shown in Figure S1A, DEPC gave the highest signal and was 43-folds higher than the next performing buffer (DEPC: $31.3 \pm 3.9$ versus IVM: $2.2 \pm 0.3$ copies/ $\mu \mathrm{L}$ Gadph DNA). The compatibility of top two performing buffers, DEPC and IVM, for direct dPCR quantification of oocytes was then verified. In DEPC, $55.1 \pm 8.6$ copies/ $\mu \mathrm{L}$ of Gadph was detected compared to $26.7 \pm 7.1$ copies/ $\mu$ L in IVM (Figure S1B). Sensitivity of the assay was next evaluated. In serially diluted lung tissue cDNA, a linearity at $r^{2}=0.999$ across a concentration range over at least three orders of magnitude from $0.1 \mathrm{ng} / \mu \mathrm{L}$ to $10 \mathrm{ng} / \mu \mathrm{L}$ (or 0.65 to 33 copies $/ \mu \mathrm{L}$ ) was achieved (Table S3A). The lower limit of detection (LLoD) of dPCR was comparable to qPCR, the 'gold standard' of genetic quantification $(0.1 \mathrm{ng} / \mu \mathrm{L}$ or 0.65 copies $/ \mu \mathrm{L}, r^{2}=0.998$, as determined by the $C_{t}$ value $>30$; Figures $2 A, 2 B$ ). Only signals generated in the partitions that separated were above the threshold of background were taken as positive signals (Figure S2). Using ATPase 6, the direct dPCR LLoD was 0.77 copies (Figure $\mathbf{2 C}$ ), verifying our sensitivity assessment of the assay. Next, the accuracy of $\mathrm{dPCR}$ in quantifying absolute gene copies of target genes was assessed by comparing spiked E. coli GapA gene of known gene copies (expected) against the dPCR readouts (observed). In a tested range of $112.5-1800$ copies/ $\mu \mathrm{L}$, the observed versus expected GapA gene copies correlated significantly $\left(r^{2}=0.999\right)$ with a gradient $(m)$ of 0.969 and demonstrated excellent reproducibility of $<10 \%$ relative standard deviation (Figure 2D; Table S3B). To circumvent the need for DNA isolation and instead, perform direct dPCR analysis, we optimized chemical-free lysis methods. Using GAPDH as the reference gene, we observed that 10 quick freeze-thaw cycles of oocytes in liquid $\mathrm{N}_{2}$ performed the best compared to 10 slow freeze-thaw cycles and one freeze-thaw lysis (data not shown). Altogether, we optimized the $\mathrm{dPCR}$ method for single oocyte analysis, determined DEPC as the buffer of choice, demonstrated accuracy in detecting bona fide absolute gene copies using $\mathrm{dPCR}$, and established the sensitivity limits of the $\mathrm{dPCR}$ assay.

\section{Direct digital mRNA analysis of individual oocytes reveals individualized genetic} makeup 
Having optimized the direct dPCR assay, we tested the assay to perform absolute quantification in single GV oocytes. From the ovaries of five mice, DNA quantification by direct dPCR showed an average of 1067.85 copies/oocyte of ATPase 6, 1.20 copies/oocyte of BubR1, 5.10 copies/oocyte of Emil1, 27.90 copies/oocyte of Pttg1, 88.20 copies/oocyte of Zp3 and 131.40 copies/oocyte of Gapdh (Figure 3A). We verified oocyte lysis by microscopy. Additionally, we rationalized that if an intact oocyte was analysed there would be no amplification. Transcript levels of Gapdh and Hprt1, commonly used housekeeping genes [22], were measured from GV oocytes obtained from the ovaries of five mice. Measured mRNA copies varied widely, even within ovaries from the same mouse (Gapdh: 0.72-16.95 copies/oocyte, range: 16.23 copies/oocyte; Hprt1: 1.05-19.05 copies/oocyte, range: 18 copies/oocyte; Figure 3B). ATPase 6, which has been shown to be highly induced in metaphase II oocytes compared to GV oocytes [35,36], also varied widely from GV oocyte to GV oocyte (5.55-32358.15 copies/oocyte, range: 32352.6 copies/oocyte; Figure $\mathbf{3 C}$ ). Therefore, absolute quantification of these expressed genes revealed individual oocyte natural variation.

\section{Diverse microRNA-mRNA interactions coordinate $C_{24: 1-C e r}$ oocyte induced maturation inhibition}

We extended the dPCR method to probing the extracellular miRNAs (exmiRNome) secreted by oocytes. $\mathrm{C}_{24: 1}$-Cer increases mitochondrial reactive oxygen species which leads to obtund oocyte maturation rates [28]. Poor maturation of oocytes from MI to MII may be associated with suboptimal interactions of the exmiRNA from poorly matured oocytes with themselves, their microenvironment and surrounding cells [37]. Among 940 miRNAs that were profiled, a total of 7 exmiRNAs were upregulated and 198 downregulated exmiRNAs in $\mathrm{C}_{24: 1}$-Cer treated murine oocytes compared to untreated oocytes ( $\log _{2}$ fold change $>2$ or $<-2$, $p$-value $<0.05$ ) (Figure 4A; Table S4). Among these, three exmiRNAs miR-380-5p, mmu-miR-1969, and mmu-miR-509-5p were selected for validation by dPCR. Significant reductions in levels of two exmiRNAs, mmu-miR-1969 and mmu-miR-509-5p, were validated by dPCR and their copy numbers quantified in the culture media from an independent set of oocytes (Figure 4B).

To further understand the potential roles of exmiRNA with $\mathrm{C}_{24: 1}$-Cer exposure, we investigated oocyte miRNA-mRNA target associations. Oocyte transcriptomes were sequenced, and paired miRNA-mRNA profiles were integrated. Two of the validated exmiRNAs, mmu-miR-1969 and mmu-miR-509-5p, were predicted to bind to Bcl2 modifying factor, $b m f$, a member of the BCL2 family that act as apoptosis regulators. In 
addition, mmu-miR-509-5p was predicted to bind to and modulate SIc22a27, a sodiumindependent organic anion transporter which exhibits high specificity for L-carnitine, a metabolite that improves oocyte maturation when supplemented in culture [38]. Six miRNAs (mmu-miR-3083-5p, mmu-miR-466I-3p, mmu-miR-883a-5p, mmu-miR-15b-5p, $m m u-m i R-188-3 p$ and $m m u-m i R-326-5 p$ ) with over ten negatively associated target genes (Table 1), consistent with the expected degradation of target mRNAs and/or inhibiting their translation. The target genes participated in different GO biological processes (BPs) or pathways, suggesting that these miRNAs have versatile regulatory roles in oocyte maturation by targeting diverse genes. KEGG enrichment analysis of miRNA-mRNA association revealed glycosphingolipid biosynthesis, fatty acid metabolism, amino acid metabolism and glycan metabolism (Table 1). Interestingly, six viral disease-associated pathways were enriched in mmu-miR-466l-3p. Upon further analysis, all pathways had a shared gene, Eif2ak2, that play key roles in regulating signal transduction of apoptosis, cell proliferation and differentiation (Figure 4C) [39]. Therefore, the results suggest Eif2ak2-associated proliferation and apoptotic processes rather than a viral response in $\mathrm{C}_{24: 1-\mathrm{Cer}}$ obtunded oocytes. Collectively, diverse miRNA-mRNA interactions coordinate the effects $\mathrm{C}_{24: 1}$-Cer on obtunding oocyte maturation. 


\section{DISCUSSION}

A high-quality oocyte is a critical prerequisite for successful fertilization and implantation, profoundly suggesting that fertility outcomes might be based on the oocyte variation. Single cell genetic analysis is increasingly investigated across different platforms because of the critical information divulged at the individual cell level [40-43]. In this study, we demonstrate direct $\mathrm{dPCR}$ analysis of DNA and RNA from single oocytes without the need for an isolation step, removing the need for extraction or purification of genetic material employed by most techniques $[22,40,44-46]$. The direct dPCR method was then applied to investigate six genes related to oocyte maturation. In addition to revealing heterogeneous expression of these genes in individual oocytes, this approach provided miRNA-target patterns of how $\mathrm{C}_{24: 1}$-Cer, a very long chained ceramide previously implicated in endometriosis-associated infertility [28], influences the oocyte transcriptome and prevent oocyte maturation.

In conventional qPCR, housekeeping genes are used for normalization purposes of the targeted gene across different samples, treatment groups or conditions [47-49]. However, the utility of these genes for normalization has been questioned given possible different gene copies varying across samples and conditions [22,50-52]. One major advantage of $\mathrm{dPCR}$ is its absolute and precise quantification. In our study, using dPCR we also showed strong correlation between what was measured and what was in the sample. Standard qPCR protocols involve the use of varying amounts of inhibitory substances, consequently leading to quantification inaccuracies and hampering inter-laboratory comparability of data [24]. In contrast, dPCR is more resistant to inhibitory effects of different matrices and is not dependent on calibration and its commutability [53]. Single sperm analysis using a different $\mathrm{dPCR}$ platform has been previously reported [54]. In addition, direct dPCR analysis of unpurified plasma or serum has been reported [21]. Major assay advantages in the Clarity ${ }^{\mathrm{TM}}$ system used in this study compared with Fluidigm system used in the sperm analysis [54] include reduction in time to analyze samples ( $<4 \mathrm{~h}$ versus $>250 \mathrm{~h}$ for analysis of 96 reactions), therefore facilitating bona fide high-throughput, and reduction in cost. Our assay also allows for straight-forward porting and adaptation of existing qRTPCR protocols from already established laboratory-customized protocols to systems such as the Clarity ${ }^{\mathrm{TM}} \mathrm{dPCR}$ platform used herein, as well as QuantStudio 3D Digital PCR System, QIAcuity Digital PCR System, and Droplet Digital PCR System. This is critical as laboratories are likely to have optimized their specific protocols, and easy portability will be favoured. In combination with the absolute and accurate quantification offered by dPCR, rapid and direct detection and quantification of genomic material without minimal loss of 
sample material and broad applicability is therefore an attractive method for evaluating oocyte quality.

An important observation of this study is the varying amounts of six genes involved in oocyte maturation in individual oocytes. While the maternal transcriptome is reported to be inactive during oogenesis and maturation and reactivate after fertilization, our results are consistent with other studies that certain loci in mouse and Drosophila are transcriptome active [55-57]. A high-quality oocyte is a critical prerequisite for successful fertilization and implantation, profoundly suggesting that fertility outcomes might be associated with oocyte variation. Furthermore, it has been shown that the phenotype of offspring are considerably defined by the quality of the oocytes from which they are derived [55-57]. The variability of genetic material copies of oocytes from the ovaries of the same mouse as shown in this study exemplifies the need to assess oocytes individually. The ability to conduct accurate and sensitive oocyte genetic analysis is critical for gaining insights to oocyte maturation, development, aging, and oocyte production in vitro, serving as a basis for understanding infertility disorders, determining fertility outcomes [58]. In addition, our findings underpin the importance of oocyte selection for assisted reproductive techniques or ART, whereby multiple oocytes are harvested from which one oocyte would be selected for artificial insemination, paving the way for increasing the chance for implantation [59].

Extracellular miRNAs are emerging as signalling molecules and raises the prospect of oocyte-to-oocyte or oocyte-to-neighbouring cell communications such as thecal cells for the development of oocytes. Many target mRNAs carry multiple miRNA sites that overlap or are in close vicinity. Binding of a miRNA to a target site may be either enhanced or repressed by the changing miRNA milieu [60]. Prominent miRNAs that were significantly altered with exogeneous $\mathrm{C}_{24: 1}$-Cer affected in oocyte maturation (Table S4) is consistent with other studies. miR-15b is known to target genes involved in cell cycle regulation and cell death $[61,62]$. The upregulation of $m i R-15 b-5 p$ is implicated in the development of ovarian toxicity [63]. Furthermore, $m i R-15 b-5 p$ has been shown to target estrogen-related receptor gamma (Esrrg). Decreased expression of $m i R-15 b$ has been associated with the production of mitochondrial-associated ROS [64], congruent with the increase of mitochondrial ROS induced by $\mathrm{C}_{24: 1}-\mathrm{Cer}$ [28]. Freudzon et al. reported that $m m u-m i R-466 /-$ $3 p$ targets GPR3 gene which maintains meiotic arrest in oocytes [65]. The downregulation of Gpr3 results in premature ovarian aging [66]. In human follicular fluids of mature oocytes, miR-380-5p is one of the more abundant miRNAs [16]. Validated decrease in miR-380-5p in our study plausible reflects oocyte maturation inhibition and suggest that $\mathrm{C}_{24: 1}$-Cer leads 
to $m i R-380-5 p$ downregulation and hence oocyte maturation inhibition. Therefore, miR380-5p offers the possibility of non-invasively selecting the most competent oocyte based on selected miRNA quantification, although further validation will be required.

Future work includes broad mRNA profiling of oocytes to further evaluate degree of oocyte transcriptome heterogeneity and the impact of oocyte transcriptome upon $\mathrm{C}_{24: 1}$-Cer exposure. Quality and integrity attributes of oocytes and reliable readouts of single oocytes are critical requisites for quantitative analysis of oocyte quality if such a method would to be employed clinically, which will inadvertently impact the success of ART. The identification and validation of exmiRNAs correlated to oocyte maturation in mouse and humans, including miR-380-5p, will add confidence to their use as non-invasive biomarkers of oocyte quality. Digital CRISPR, which was also developed on the Clarity dPCR platform [67] can be evaluated and considered for improved detection of exmiRNA in oocytes.

In conclusion, our study optimized a dPCR protocol that can be performed in $3 \mathrm{~h}$, and demonstrated the feasibility to quantify mRNA from single oocytes without the need for sample extraction or purification. Further, we demonstrated the non-invasive application of $\mathrm{dPCR}$ in quantifying exmiRNAs oocyte culture media [28], paving the possibility of investigating extracellular miRNAs from blastocysts and embryos [68] but at the single cell level.

\section{ACKNOWLEDGEMENTS}

This work was supported by SingHealth Foundation (SHF/FG560P/2014) and National Medical Research Council, Singapore (NMRC/BNIG/2033/2015).

\section{REFERENCES}

[1] C. De Geyter, More than 8 million babies born from IVF since the world's first in 1978, Eur. Soc. Hum. Reprod. Embryol. (2018).

[2] C. Gnoth, B. Maxrath, T. Skonieczny, K. Friol, E. Godehardt, J. Tigges, Final ART success rates: a 10 years survey, Hum. Reprod. 26 (2011) 2239-2246. https://doi.org/10.1093/humrep/der178.

[3] W. Schoolcraft, M. Meseguer, Paving the way for a gold standard of care for infertility treatment: improving outcomes through standardization of laboratory procedures, Reprod. Biomed. Online. 35 (2017) 391-399. 
https://doi.org/10.1016/j.rbmo.2017.06.023.

[4] N. Hamazaki, H. Kyogoku, H. Araki, F. Miura, C. Horikawa, N. Hamada, S. Shimamoto, O. Hikabe, K. Nakashima, T.S. Kitajima, T. Ito, H.G. Leitch, K. Hayashi, Reconstitution of the oocyte transcriptional network with transcription factors, Nature. 589 (2021) 264-269. https://doi.org/10.1038/s41586-020-3027-9.

[5] T.C. Thomson, K.E. Fitzpatrick, J. Johnson, Intrinsic and extrinsic mechanisms of oocyte loss, Mol. Hum. Reprod. 16 (2010) 916-927. https://doi.org/10.1093/molehr/gaq066.

[6] Y. Hou, W. Fan, L. Yan, R. Li, Y. Lian, J. Huang, J. Li, L. Xu, F. Tang, X.S. Xie, J. Qiao, Genome Analyses of Single Human Oocytes, Cell. 155 (2013) 1492-1506. https://doi.org/10.1016/j.cell.2013.11.040.

[7] N.R. Mtango, S. Potireddy, K.E. Latham, Chapter 7 Oocyte Quality and Maternal Control of Development, in: 2008: pp. 223-290. https://doi.org/10.1016/S19376448(08)00807-1.

[8] M. Yáñez-Mó, P.R.-M. Siljander, Z. Andreu, A. Bedina Zavec, F.E. Borràs, E.I. Buzas, K. Buzas, E. Casal, F. Cappello, J. Carvalho, E. Colás, A. Cordeiro-da Silva, S. Fais, J.M. Falcon-Perez, I.M. Ghobrial, B. Giebel, M. Gimona, M. Graner, I. Gursel, M. Gursel, N.H.H. Heegaard, A. Hendrix, P. Kierulf, K. Kokubun, M. Kosanovic, V. Kralj-Iglic, E.-M. Krämer-Albers, S. Laitinen, C. Lässer, T. Lener, E. Ligeti, A. Linē, G. Lipps, A. Llorente, J. Lötvall, M. Manček-Keber, A. Marcilla, M. Mittelbrunn, I. Nazarenko, E.N.M. Nolte-‘t Hoen, T.A. Nyman, L. O’Driscoll, M. Olivan, C. Oliveira, É. Pállinger, H.A. del Portillo, J. Reventós, M. Rigau, E. Rohde, M. Sammar, F. Sánchez-Madrid, N. Santarém, K. Schallmoser, M. Stampe Ostenfeld, W. Stoorvogel, R. Stukelj, S.G. Van der Grein, M. Helena Vasconcelos, M.H.M. Wauben, O. De Wever, Biological properties of extracellular vesicles and their physiological functions, J. Extracell. Vesicles. 4 (2015) 27066. https://doi.org/10.3402/jev.v4.27066.

[9] D.S. J.C., S. D.R., V. D.N.R., W. Q.A., C. E.M., B. G.J., MiRNAs within the ovarian follicle: Identification of cell-secreted vesicles as miRNA carriers, Biol. Reprod. (2011).

[10] R. Machtinger, L.C. Laurent, A.A. Baccarelli, Extracellular vesicles: roles in gamete maturation, fertilization and embryo implantation, Hum. Reprod. Update. (2015) dmv055. https://doi.org/10.1093/humupd/dmv055.

[11] H. Guo, N.T. Ingolia, J.S. Weissman, D.P. Bartel, Mammalian microRNAs predominantly act to decrease target mRNA levels, Nature. (2010). https://doi.org/10.1038/nature09267.

[12] N. Bushati, S.M. Cohen, microRNA functions., Annu. Rev. Cell Dev. Biol. 23 
(2007) 175-205. https://doi.org/10.1146/annurev.cellbio.23.090506.123406.

[13] B. Pan, D. Toms, J. Li, MicroRNA-574 suppresses oocyte maturation via targeting hyaluronan synthase 2 in porcine cumulus cells, Am. J. Physiol. - Cell Physiol. (2018). https://doi.org/10.1152/ajpcell.00065.2017.

[14] R. Battaglia, M.E. Vento, M. Ragusa, D. Barbagallo, A. La Ferlita, G. Di Emidio, P. Borzi, P.G. Artini, P. Scollo, C. Tatone, M. Purrello, C. Di Pietro, MicroRNAs Are Stored in Human MII Oocyte and Their Expression Profile Changes in Reproductive Aging, Biol. Reprod. 95 (2016) 131-131. https://doi.org/10.1095/biolreprod.116.142711.

[15] R. Pasquariello, B. Badaoui, A. Ermisch, E.E. Paulson, S. McCormick, J.P. Barfield, W.B. Schoolcraft, P.J. Ross, R.L. Krisher, Micro-RNA sequencing of individual human oocytes, Fertil. Steril. 108 (2017) e144. https://doi.org/10.1016/j.fertnstert.2017.07.435.

[16] R. Machtinger, R.S. Rodosthenous, M. Adir, A. Mansour, C. Racowsky, A.A. Baccarelli, R. Hauser, Extracellular microRNAs in follicular fluid and their potential association with oocyte fertilization and embryo quality: an exploratory study, J. Assist. Reprod. Genet. (2017). https://doi.org/10.1007/s10815-017-0876-8.

[17] P.J. Sykes, S.H. Neoh, M.J. Brisco, E. Hughes, J. Condon, A.A. Morley, Quantitation of targets for PCR by use of limiting dilution, Biotechniques. (1992). https://doi.org/10.1177/0363546512467952.

[18] B. Vogelstein, K.W. Kinzler, Digital PCR, Proc. Natl. Acad. Sci. (1999). https://doi.org/10.1073/pnas.96.16.9236.

[19] A.A. Morley, Digital PCR: A brief history, Biomol. Detect. Quantif. (2014). https://doi.org/10.1016/j.bdq.2014.06.001.

[20] R.A. White, P.C. Blainey, H.C. Fan, S.R. Quake, Digital PCR provides sensitive and absolute calibration for high throughput sequencing, BMC Genomics. (2009). https://doi.org/10.1186/1471-2164-10-116.

[21] D. Sefrioui, L. Beaussire, A. Perdrix, F. Clatot, P. Michel, T. Frebourg, F. Di Fiore, N. Sarafan-Vasseur, Direct circulating tumor DNA detection from unpurified plasma using a digital PCR platform, Clin. Biochem. 50 (2017) 963-966. https://doi.org/10.1016/j.clinbiochem.2017.06.005.

[22] S. Mamo, A.B. Gal, S. Bodo, A. Dinnyes, Quantitative evaluation and selection of reference genes in mouse oocytes and embryos cultured in vivo and in vitro, BMC Dev. Biol. (2007). https://doi.org/10.1186/1471-213X-7-14.

[23] F. Jacob, R. Guertler, S. Naim, S. Nixdorf, A. Fedier, N.F. Hacker, V. Heinzelmann-Schwarz, Careful Selection of Reference Genes Is Required for Reliable Performance of RT-qPCR in Human Normal and Cancer Cell Lines, 
PLoS One. 8 (2013) e59180. https://doi.org/10.1371/journal.pone.0059180.

[24] T. Demeke, G.R. Jenkins, Influence of DNA extraction methods, PCR inhibitors and quantification methods on real-time PCR assay of biotechnology-derived traits, Anal. Bioanal. Chem. 396 (2010) 1977-1990. https://doi.org/10.1007/s00216-009-3150-9.

[25] L. Cohen, M.R. Hartman, A. Amardey-Wellington, D.R. Walt, Digital direct detection of microRNAs using single molecule arrays, Nucleic Acids Res. 45 (2017) e137-e137. https://doi.org/10.1093/nar/gkx542.

[26] H. Low, S.J. Chan, G.H. Soo, B. Ling, E.L. Tan, Clarity ${ }^{T M}$ digital PCR system: a novel platform for absolute quantification of nucleic acids, Anal. Bioanal. Chem. (2017). https://doi.org/10.1007/s00216-016-0131-7.

[27] J.H. Vo, W.L. Nei, M. Hu, W.M. Phyo, F. Wang, K.W. Fong, T. Tan, Y.L. Soong, S.L. Cheah, K. Sommat, H. Low, B. Ling, J. Ng, W.L. Tan, K.S. Chan, L. Oon, J.Y. Ying, M.H. Tan, Comparison of Circulating Tumour Cells and Circulating Cell-Free Epstein-Barr Virus DNA in Patients with Nasopharyngeal Carcinoma Undergoing Radiotherapy, Sci. Rep. (2016). https://doi.org/10.1038/s41598-016-0006-3.

[28] Y.H. Lee, J.X. Yang, J.C. Allen, C.S. Tan, B.S.M. Chern, T.Y. Tan, H.H. Tan, C.N.Z. Mattar, J.K.Y. Chan, Elevated peritoneal fluid ceramides in human endometriosis-associated infertility and their effects on mouse oocyte maturation, Fertil. Steril. 110 (2018) 767-777.e5.

https://doi.org/10.1016/j.fertnstert.2018.05.003.

[29] M.E. Ritchie, B. Phipson, D. Wu, Y. Hu, C.W. Law, W. Shi, G.K. Smyth, limma powers differential expression analyses for RNA-sequencing and microarray studies., Nucleic Acids Res. 43 (2015) e47. https://doi.org/10.1093/nar/gkv007.

[30] L. Chen, D.L. Gibbons, S. Goswami, M.A. Cortez, Y.-H. Ahn, L.A. Byers, X. Zhang, X. Yi, D. Dwyer, W. Lin, L. Diao, J. Wang, J. Roybal, M. Patel, C. Ungewiss, D. Peng, S. Antonia, M. Mediavilla-Varela, G. Robertson, M. Suraokar, J.W. Welsh, B. Erez, I.I. Wistuba, L. Chen, D. Peng, S. Wang, S.E. Ullrich, J. V Heymach, J.M. Kurie, F.X.-F. Qin, Metastasis is regulated via microRNA200/ZEB1 axis control of tumour cell PD-L1 expression and intratumoral immunosuppression., Nat. Commun. 5 (2014) 5241. https://doi.org/10.1038/ncomms6241.

[31] H. Dweep, N. Gretz, miRWalk2.0: a comprehensive atlas of microRNA-target interactions., Nat. Methods. 12 (2015) 697. https://doi.org/10.1038/nmeth.3485.

[32] M. Ashburner, C.A. Ball, J.A. Blake, D. Botstein, H. Butler, J.M. Cherry, A.P. Davis, K. Dolinski, S.S. Dwight, J.T. Eppig, M.A. Harris, D.P. Hill, L. Issel-Tarver, A. Kasarskis, S. Lewis, J.C. Matese, J.E. Richardson, M. Ringwald, G.M. Rubin, 
G. Sherlock, Gene ontology: tool for the unification of biology. The Gene Ontology Consortium., Nat. Genet. 25 (2000) 25-9. https://doi.org/10.1038/75556.

[33] M. Kanehisa, S. Goto, KEGG: kyoto encyclopedia of genes and genomes., Nucleic Acids Res. 28 (2000) 27-30.

http://www.ncbi.nlm.nih.gov/pubmed/10592173.

[34] P. Shannon, A. Markiel, O. Ozier, N.S. Baliga, J.T. Wang, D. Ramage, N. Amin, B. Schwikowski, T. Ideker, Cytoscape: a software environment for integrated models of biomolecular interaction networks., Genome Res. 13 (2003) 2498-504. https://doi.org/10.1101/gr.1239303.

[35] X.S. Cui, H. Song, N.H. Kim, Identification of metaphase II-specific gene transcripts in porcine oocytes and their expression in early stage embryos, Reprod. Fertil. Dev. (2005). https://doi.org/10.1071/RD05019.

[36] S. Markholt, M.L. Grøndahl, E.H. Ernst, C.Y. Andersen, E. Ernst, K. LykkeHartmann, Global gene analysis of oocytes from early stages in human folliculogenesis shows high expression of novel genes in reproduction, Mol. Hum. Reprod. (2012). https://doi.org/10.1093/molehr/gar083.

[37] S.W. Maalouf, W.S. Liu, J.L. Pate, MicroRNA in ovarian function, Cell Tissue Res. 363 (2016) 7-18. https://doi.org/10.1007/s00441-015-2307-4.

[38] A. Agarwal, P. Sengupta, D. Durairajanayagam, Role of L-carnitine in female infertility, Reprod. Biol. Endocrinol. 16 (2018) 5. https://doi.org/10.1186/s12958018-0323-4.

[39] M.A. Garcia, J. Gil, I. Ventoso, S. Guerra, E. Domingo, C. Rivas, M. Esteban, Impact of Protein Kinase PKR in Cell Biology: from Antiviral to Antiproliferative Action, Microbiol. Mol. Biol. Rev. 70 (2006) 1032-1060. https://doi.org/10.1128/MMBR.00027-06.

[40] N. Steuerwald, J. Cohen, R.J. Herrera, C.A. Brenner, Quantification of mRNA in single oocytes and embryos by real-time rapid cycle fluorescence monitored RTPCR, Mol. Hum. Reprod. (2000). https://doi.org/10.1093/molehr/6.5.448.

[41] S.S.-L. Li, Y.-H. Liu, C.-N. Tseng, S. Singh, Analysis of gene expression in single human oocytes and preimplantation embryos, Biochem. Biophys. Res. Commun. (2006). https://doi.org/10.1016/j.bbrc.2005.11.149.

[42] M.G. Bermúdez, D. Wells, H. Malter, S. Munné, J. Cohen, N.M. Steuerwald, Expression profiles of individual human oocytes using microarray technology, Reprod. Biomed. Online. (2004). https://doi.org/10.1016/S1472-6483(10)60913-3.

[43] A. Reich, P. Klatskys, S. Carson, G. Wessel, The transcriptome of a human polar body accurately reflects its sibling oocyte, J. Biol. Chem. (2011).

https://doi.org/10.1074/jbc.M111.289868. 
[44] M. Poli, A. Ori, T. Child, S. Jaroudi, K. Spath, M. Beck, D. Wells, Characterization and quantification of proteins secreted by single human embryos prior to implantation, EMBO Mol. Med. (2015). https://doi.org/10.15252/emmm.201505344.

[45] J.C. Parks, B.R. McCallie, A.M. Janesch, W.B. Schoolcraft, M.G. Katz-Jaffe, Blastocyst gene expression correlates with implantation potential, Fertil. Steril. (2011). https://doi.org/10.1016/j.fertnstert.2010.08.009.

[46] E. Scalici, S. Traver, T. Mullet, N. Molinari, A. Ferrières, C. Brunet, S. Belloc, S. Hamamah, Circulating microRNAs in follicular fluid, powerful tools to explore in vitro fertilization process, Sci. Rep. (2016). https://doi.org/10.1038/srep24976.

[47] D. Poel, T.E. Buffart, J. Oosterling-Jansen, H.M.W. Verheul, J. Voortman, Evaluation of several methodological challenges in circulating miRNA qPCR studies in patients with head and neck cancer, Exp. Mol. Med. (2018). https://doi.org/10.1038/emm.2017.288.

[48] S. Nielsen, N. Bassler, L. Grzanka, J. Swakon, P. Olko, C.N. Andreassen, J. Alsner, B.S. Sørensen, Optimal reference genes for normalization of qPCR gene expression data from proton and photon irradiated dermal fibroblasts, Sci. Rep. (2018). https://doi.org/10.1038/s41598-018-30946-0.

[49] D. Ling, P.M. Salvaterra, Robust RT-qPCR data normalization: Validation and selection of internal reference genes during post-experimental data analysis, PLoS One. (2011). https://doi.org/10.1371/journal.pone.0017762.

[50] C. Rubie, K. Kempf, J. Hans, T. Su, B. Tilton, T. Georg, B. Brittner, B. Ludwig, M. Schilling, Housekeeping gene variability in normal and cancerous colorectal, pancreatic, esophageal, gastric and hepatic tissues, Mol. Cell. Probes. 19 (2005) 101-109. https://doi.org/10.1016/j.mcp.2004.10.001.

[51] H.K. Hamalainen, J.C. Tubman, S. Vikman, T. Kyrölä, E. Ylikoski, J.A. Warrington, R. Lahesmaa, Identification and Validation of Endogenous Reference Genes for Expression Profiling of T Helper Cell Differentiation by Quantitative Real-Time RTPCR, Anal. Biochem. 299 (2001) 63-70. https://doi.org/10.1006/abio.2001.5369.

[52] H.J. Jacob, K. Abrams, D.P. Bick, K. Brodie, P. Dimmock, M. Farrell, J. Geurts, J. Harris, B.J. Joers, R. Kliegman, G. Kowalski, D.A. Margolis, P. North, J. Northup, G. Scharer, M. Shimoyama, B. Taylor, S. Tsaih, M.R. Tschannen, L. Veith, J. Wendt-andrae, B. Wilk, E.A. Worthey, C O M M E N TA R Y Genomics in Clinical Practice :, 5 (2013).

[53] J.F. Huggett, S. Cowen, C.A. Foy, Considerations for Digital PCR as an Accurate Molecular Diagnostic Tool, Clin. Chem. 61 (2015) 79-88.

https://doi.org/10.1373/clinchem.2014.221366. 
[54] T. Yu, C. Tang, Y. Zhang, R. Zhang, W. Yan, Microfluidics-based digital quantitative PCR for single-cell small RNA quantification†, Biol. Reprod. 97 (2017) 490-496. https://doi.org/10.1093/biolre/iox102.

[55] P. Navarro-Costa, A. McCarthy, P. Prudêncio, C. Greer, L.G. Guilgur, J.D. Becker, J. Secombe, P. Rangan, R.G. Martinho, Early programming of the oocyte epigenome temporally controls late prophase I transcription and chromatin remodelling, Nat. Commun. 7 (2016) 12331. https://doi.org/10.1038/ncomms12331.

[56] M. Almonacid, A. Al Jord, S. El-Hayek, A. Othmani, F. Coulpier, S. Lemoine, K. Miyamoto, R. Grosse, C. Klein, T. Piolot, P. Mailly, R. Voituriez, A. Genovesio, M.$\mathrm{H}$. Verlhac, Active Fluctuations of the Nuclear Envelope Shape the Transcriptional Dynamics in Oocytes, Dev. Cell. 51 (2019) 145-157.e10. https://doi.org/10.1016/j.devcel.2019.09.010.

[57] N.R. Mtango, S. Potireddy, K.E. Latham, Oocyte quality and maternal control of development, Int. Rev. Cell Mol. Biol. 268 (2008) 223-290.

[58] J.M. Stringer, P.S. Western, A step toward making human oocytes, Nat. Biotechnol. 37 (2019) 24-25. https://doi.org/10.1038/nbt.4329.

[59] S. Ozturk, Selection of competent oocytes by morphological criteria for assisted reproductive technologies, Mol. Reprod. Dev. 87 (2020) 1021-1036. https://doi.org/10.1002/mrd.23420.

[60] A. Grimson, K.K.-H. Farh, W.K. Johnston, P. Garrett-Engele, L.P. Lim, D.P. Bartel, MicroRNA targeting specificity in mammals: determinants beyond seed pairing., Mol. Cell. 27 (2007) 91-105. https://doi.org/10.1016/j.molcel.2007.06.017.

[61] G. Sun, L. Shi, S. Yan, Z. Wan, N. Jiang, L. Fu, M. Li, J. Guo, MiR-15b targets cyclin D1 to regulate proliferation and apoptosis in glioma cells, Biomed Res. Int. (2014). https://doi.org/10.1155/2014/687826.

[62] M. Rahman, F. Lovat, G. Romano, F. Calore, M. Acunzo, E.H. Bell, P. NanaSinkam, MiR-15b/16-2 regulates factors that promote p53 phosphorylation and augments the DNA damage response following radiation in the lung, J. Biol. Chem. (2014). https://doi.org/10.1074/jbc.M114.573592.

[63] H.C. Furlong, M.R. Stämpfli, A.M. Gannon, W.G. Foster, Identification of microRNAs as potential markers of ovarian toxicity, J. Appl. Toxicol. (2018). https://doi.org/10.1002/jat.3583.

[64] A. Lang, S. Grether-Beck, M. Singh, F. Kuck, S. Jakob, A. Kefalas, S. AltinolukHambüchen, N. Graffmann, M. Schneider, A. Lindecke, H. Brenden, I. Felsner, H. Ezzahoini, A. Marini, S. Weinhold, A. Vierkötter, J. Tigges, S. Schmidt, K. Stühler, K. Köhrer, M. Uhrberg, J. Haendeler, J. Krutmann, R.P. Piekorz, MicroRNA-15b 
regulates mitochondrial ROS production and the senescence-associated secretory phenotype through sirtuin 4/SIRT4, Aging (Albany. NY). (2016).

https://doi.org/10.18632/aging.100905.

[65] L. Freudzon, R.P. Norris, A.R. Hand, S. Tanaka, Y. Saeki, T.L.Z. Jones, M.M. Rasenick, C.H. Berlot, L.M. Mehlmann, L.A. Jaffe, Regulation of meiotic prophase arrest in mouse oocytes by GPR3, a constitutive activator of the Gs $G$ protein, J. Cell Biol. (2005). https://doi.org/10.1083/jcb.200506194.

[66] C. Ledent, I. Demeestere, D. Blum, J. Petermans, T. Hamalainen, G. Smits, G. Vassart, Premature ovarian aging in mice deficient for Gpr3, Proc. Natl. Acad. Sci. 102 (2005) 8922-8926. https://doi.org/10.1073/pnas.0503840102.

[67] X. Wu, J.K. Tay, C.K. Goh, C. Chan, Y.H. Lee, S.L. Springs, D.Y. Wang, K.S. Loh, T.K. Lu, H. Yu, Digital CRISPR-based method for the rapid detection and absolute quantification of nucleic acids, Biomaterials. 274 (2021) 120876.

https://doi.org/10.1016/j.biomaterials.2021.120876.

[68] A. Capalbo, F.M. Ubaldi, D. Cimadomo, L. Noli, Y. Khalaf, A. Farcomeni, D. Ilic, L. Rienzi, MicroRNAs in spent blastocyst culture medium are derived from trophectoderm cells and can be explored for human embryo reproductive competence assessment, Fertil. Steril. (2016). https://doi.org/10.1016/j.fertnstert.2015.09.014.

\section{FIGURE LEGENDS}

Figure 1. Workflow of direct digital PCR (dPCR) protocol and optimization. (A) Single oocytes were harvested, lysed by freeze-thawing, and proceed to perform (B) one-step RT-dPCR as detailed in text. The entire workflow took from oocyte freeze-thaw to image acquisition was $\sim 4 \mathrm{~h}$.

Figure 2. Direct dPCR limit of detection (LLoD). The LLoD of direct dPCR (A) was compared to the LLoD of conventional qPCR (B). Both had LLoD of $0.1 \mathrm{ng} / \mathrm{mL}$. (C) The dilution of a single oocyte was analysed using direct $\mathrm{APCR}$, showing a linear dilution curve with $\mathrm{R}^{2}$ close to 1 . By diluting a single oocyte to various ratios and detecting for ATPase6, we were similarly able to show a linear dilution curve down to very low copies of 0.77 copies/uL. The position plots on the right are the representative plots of each dilution. With each dilution, there is a corresponding decrease in the number of positive signals. It is important to note here that the number of dilution of a single oocyte would be dependent on how abundant the gene is expressed in the oocyte itself. (D) Quantification of absolute gene copies of target gene by direct $\mathrm{dPCR}$ was assessed by comparing spiked $E$. coli GapA gene of known gene copies (expected) against the $\mathrm{dPCR}$ readouts (observed). In a 
tested range of $112.5-1800$ copies/ $\mu \mathrm{L}$, the observed versus expected GapA gene copies correlated significantly $\left(r^{2}=0.999\right)$ with a gradient $(m)$ of 0.969 .

Figure 3. Natural variation of mRNA from individual GV oocytes. Direct dPCR of Gapdh, Hprt1 and ATPase 6 mRNA revealed wide variation within ovaries from the same mouse (Gapdh: 0.72-16.95 copies/oocyte, range: 16.23 copies/oocyte; Hprt1: 1.05-19.05 copies/oocyte, range: 18 copies/oocyte; ATPase 6: 5.55-32358.15 copies/oocyte, range: 32352.6 copies/oocyte). Dots of the same colour represent the oocytes obtained from the same mouse. $N=4$ mice.

Figure 4. Extracellular microRNA (exmiRNA) profiling and network biology analysis of oocytes upon $\mathbf{C}_{24: 1}$-Cer exposure. (A) Volcano plot of significantly differential exmiRNA from conditioned media of oocytes incubated with $\mathrm{C}_{24: 1}$-Cer and vehicle (control) as quantified using miRNome PCR array. 212 miRNAs were upregulated (red) and 151 miRNAs were downregulated (green) in the $\mathrm{C}_{24: 1}$-Cer treated group. (B) Decrease in miRNA 1969 and 509 were validated by dPCR from an independent set of oocytes $(N=3)$. (C) Bioinformatic analysis of miRNA-mRNA pairs of Eif2ak2, a gene that play key roles in regulating signal transduction of apoptosis, cell proliferation and differentiation.

Table 1. Gene Ontology and KEGG analysis of oocyte miRNA-mRNA associations with C24:1-ceramide 
A

B

Harvest and denude mouse oocyte

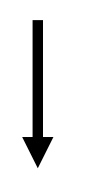

Rinse single oocyte in DEPC

$\downarrow$

Freeze-thaw single oocyte in 6 $\mu L$ DEPC

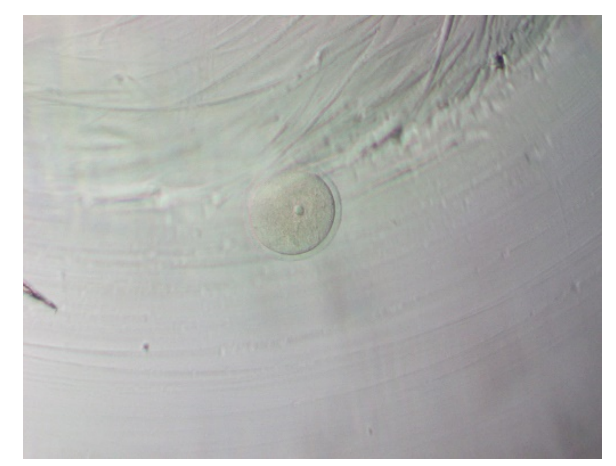

Prepare dPCR Mastermix

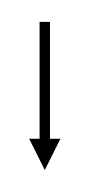

Load sample and mastermix into chip + sealing (10 min)

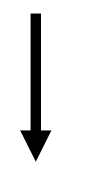

Reverse transcription + amplification (3h)

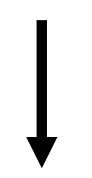

Image acquisition (8 samples/5 min)

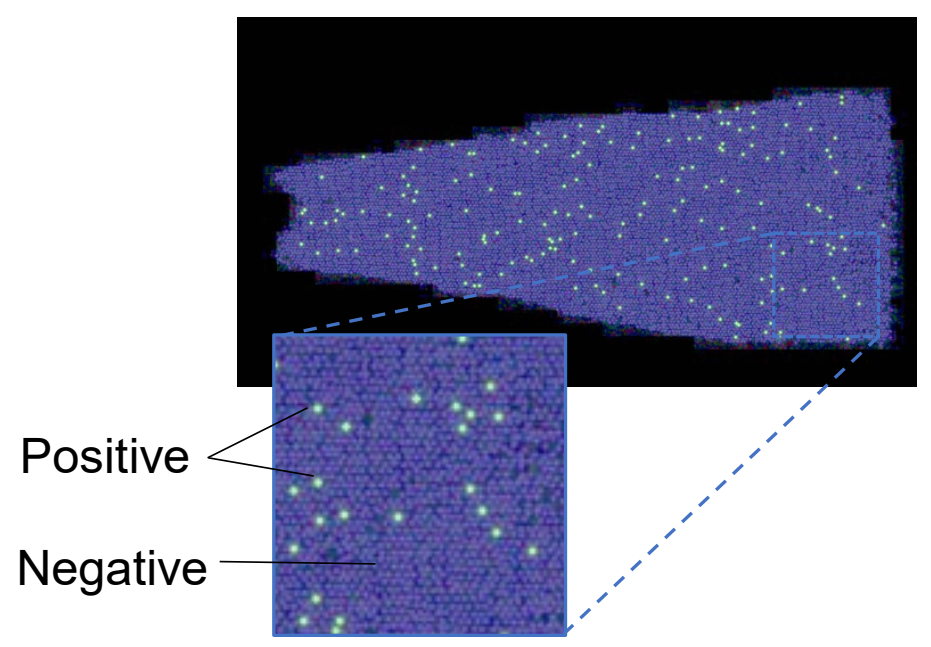


A

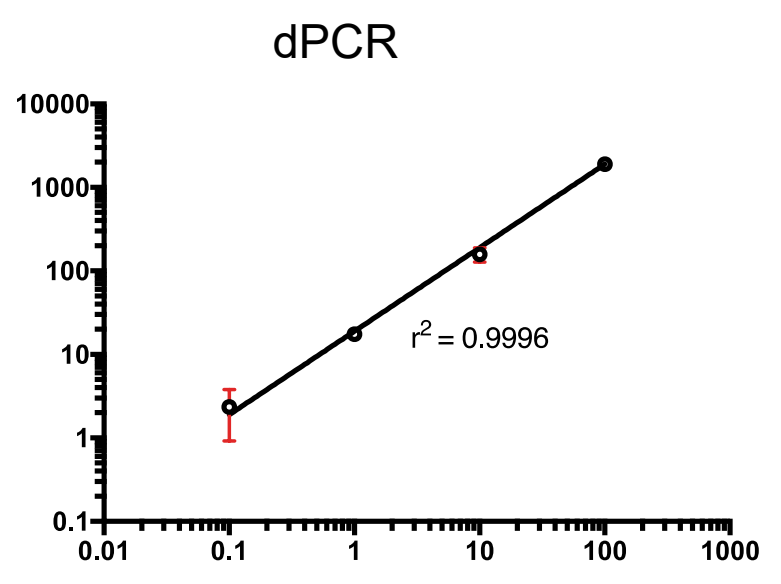

B

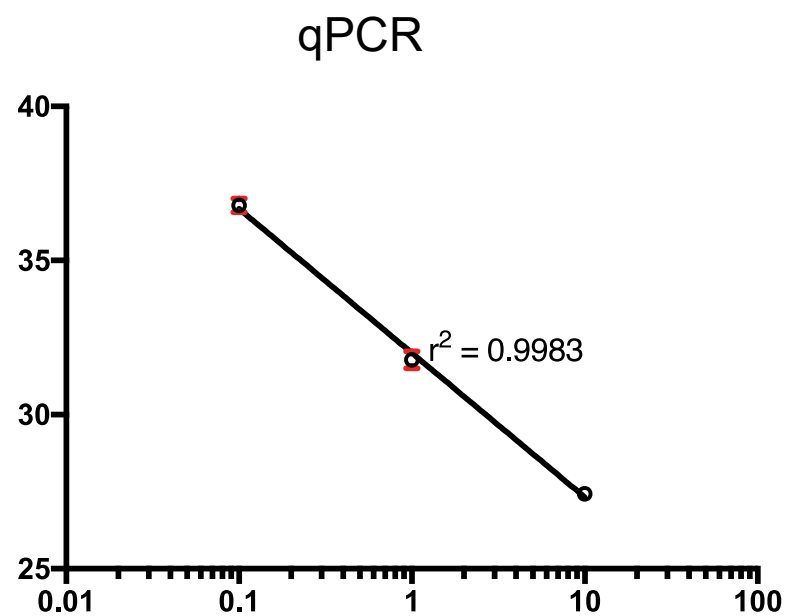

C
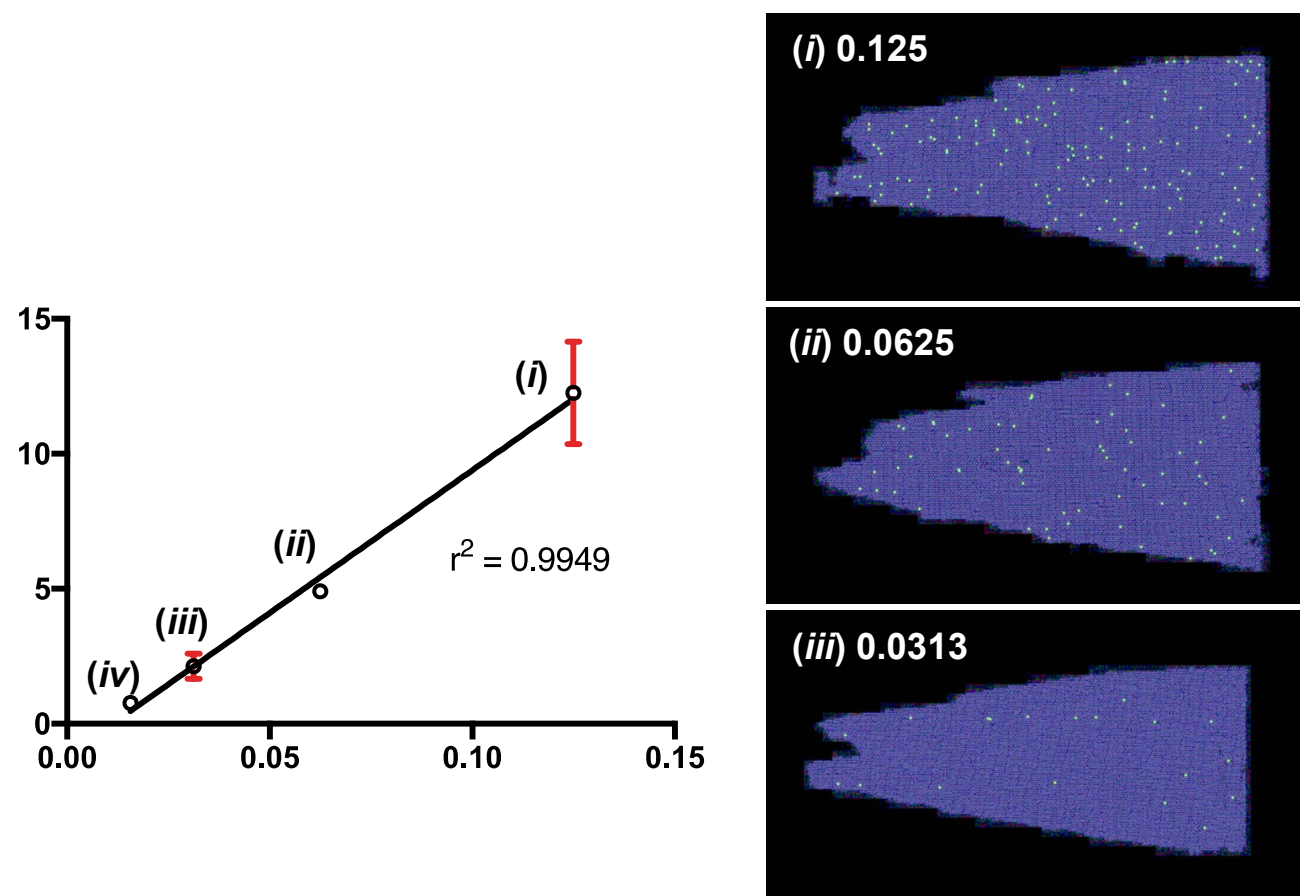

(iv) 0.0156

D

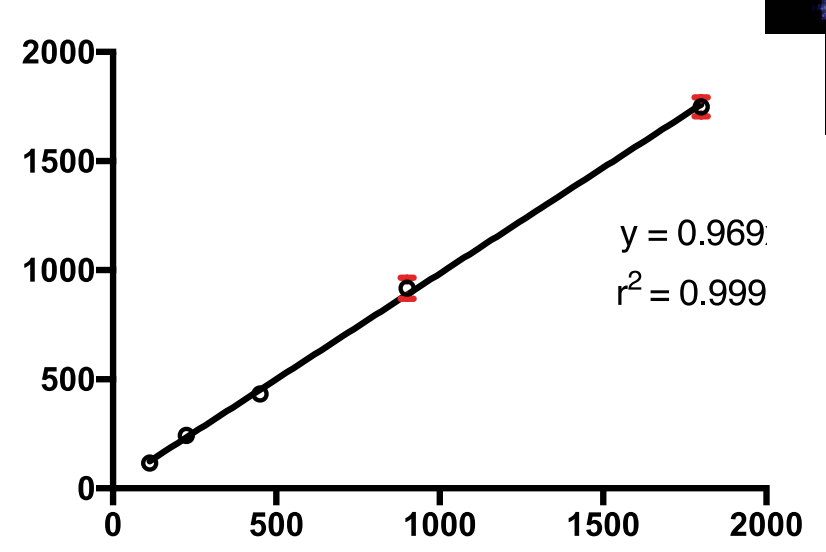


A

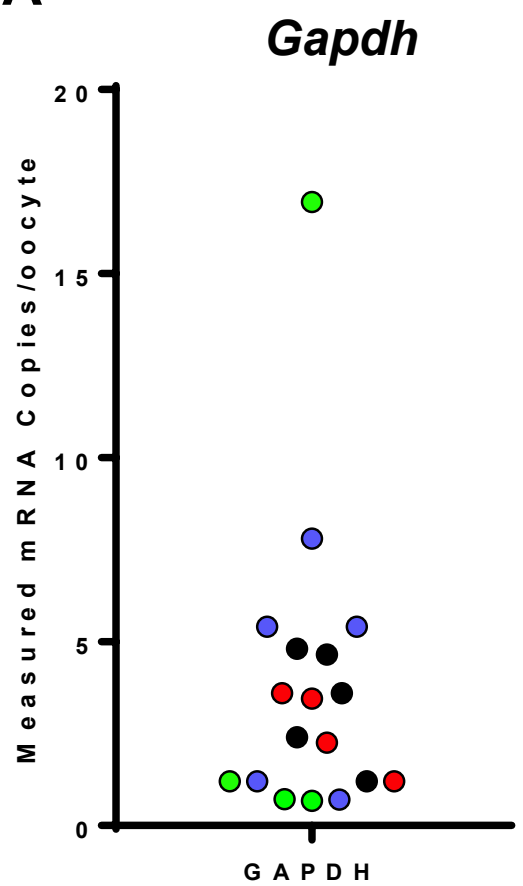

B

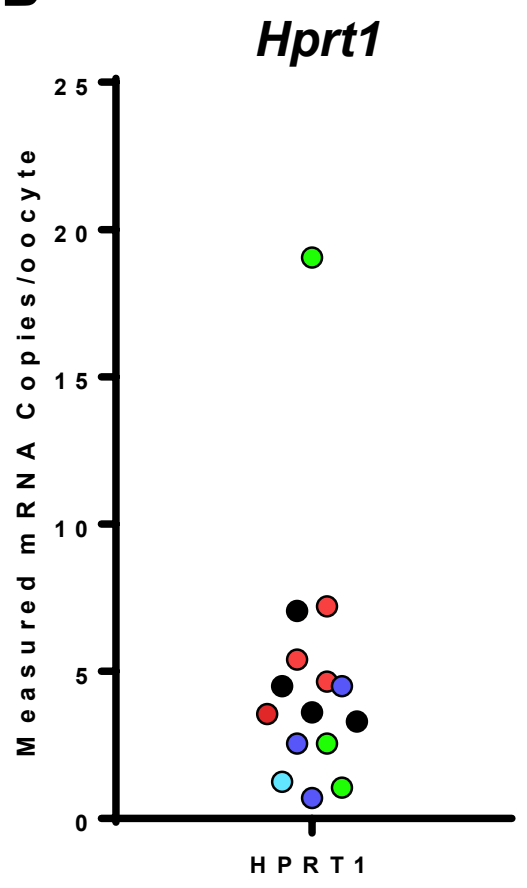

C

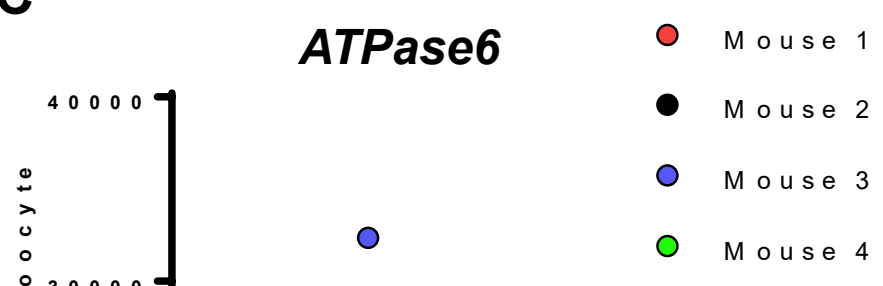




\section{FIGURE 4}

A

m iR N A

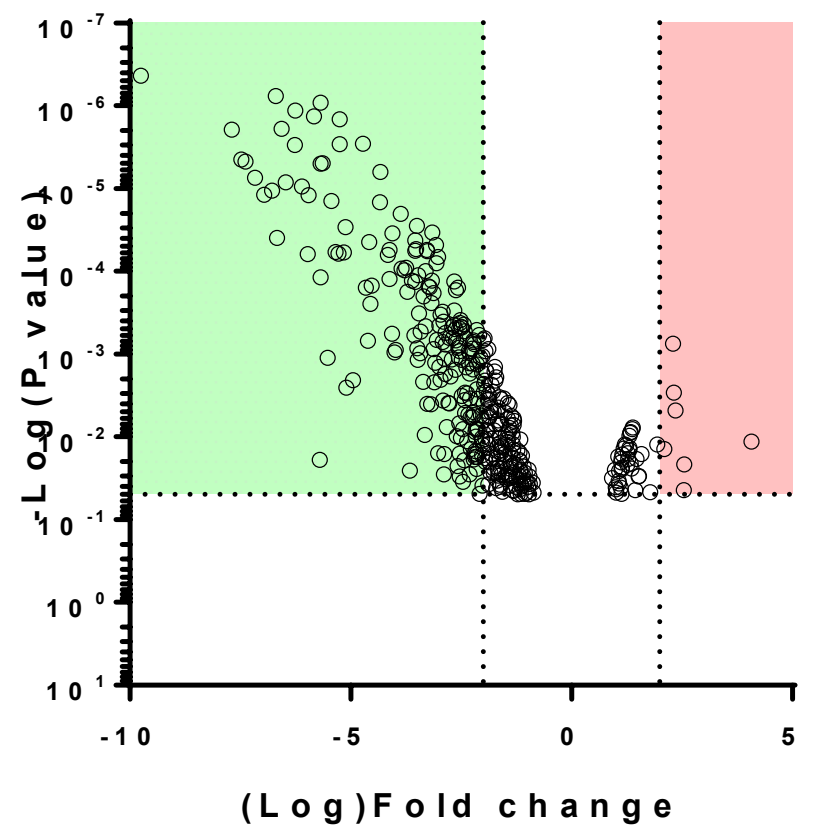

C

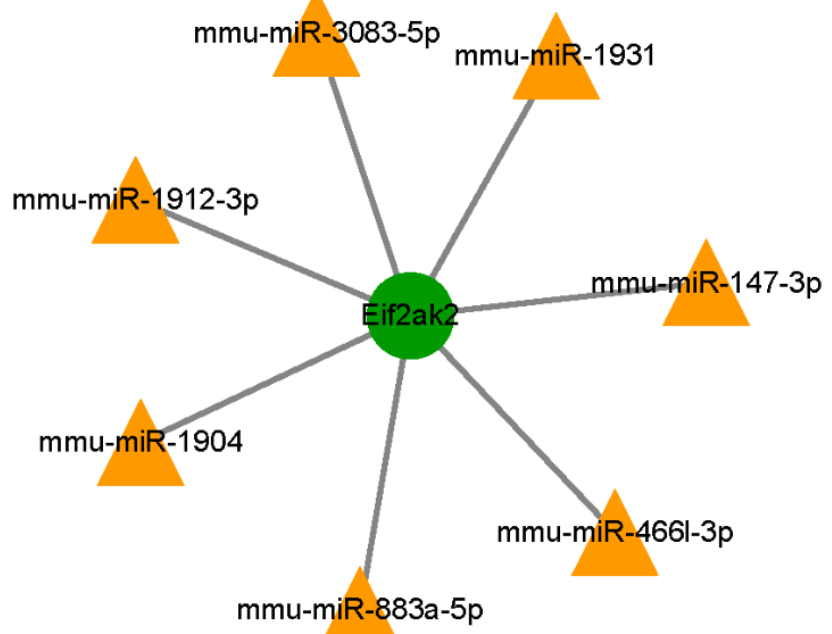

B

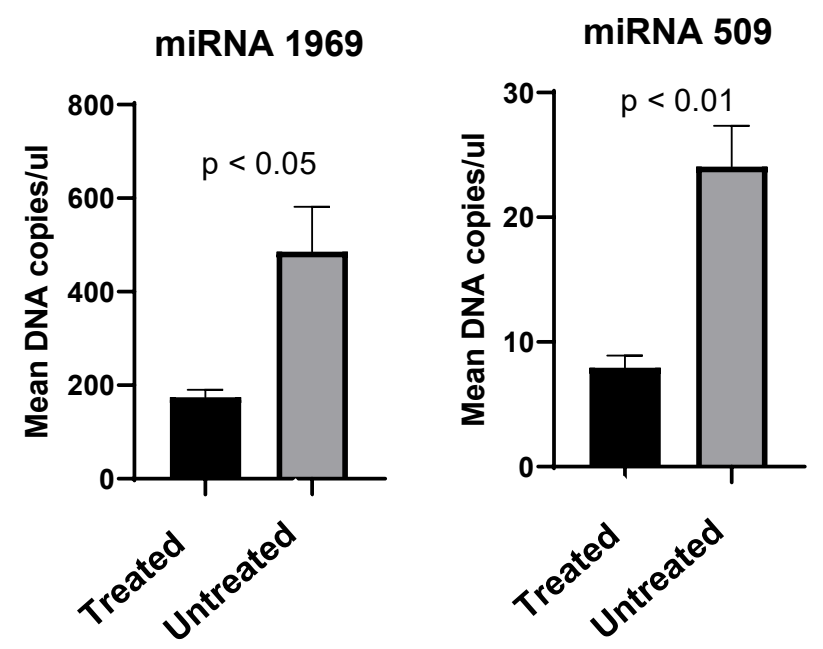




\section{miRNA}

mmu-miR-3083-5p mmu-miR-466I-3p mmu-miR-883a-5p mmu-miR-15b-5p mmu-miR-188-3p mmu-miR-326-5p

\section{Target gene}

Arhgef15 Clmn Dao Fmo2 Hexdc Hoxc13 Nlrx1 Slc13a3 Adap1 C1rl Clcnka Elovl6 Fgfr1 Gcnt1 Srcin1 Apol6 Lemd2 Shisa7 Arid5b Atf3 Cybrd1 Kcnab2 Slc35c2 Zfp185 Adrb1 Aip C530008M17Rik II31 Ptpdc1 Slc35f3 Bcorl1 Eif2ak2 H2-T24 Pappa Pknox2 Zic1 2410131K14Rik Cd8a Eif2ak2 Itga11 Aldh8a1 Asic1 Cd300a Ptcd1 Ret Tbc1d16 Tead4 Abcg4 Numa1 Slc38a4 Gramd3 Wdtc1 Gcnt1 Lipe Slc25a25 Apba1 Clmn Napa Nlrx1 Plekha6 Aifm2 Bhmt Dock2 Fmo2 Rhbdf2 Smad7 Hap1 Itga11 Lrfn2 Tspan7 Cd300a Ret St3gal1 Abcg4 Btbd16 Col26a1 Cplx2 Itga11 Tmem101 Cplx2 Ret Tubb3 Abcg4 Asic1 Cd300a Col26a1 Ptpn7 St3gal1

\section{Function analysis of the target genes}

\section{GO enrichment (Biological Process)}

\section{Cluster \\ ID}

mmu-miR-3083-5p GO:0050660

mmu-miR-3083-5p GO:0003884

mmu-miR-3083-5p GO:0004312

mmu-miR-3083-5p GO:0005247

mmu-miR-3083-5p GO:0008509

mmu-miR-3083-5p GO:0050662

mmu-miR-3083-5p GO:0015172

mmu-miR-3083-5p GO:0031404

mmu-miR-3083-5p GO:0008308

mmu-miR-3083-5p GO:0005096

mmu-miR-3083-5p GO:0071949

mmu-miR-3083-5p GO:0016641

mmu-miR-3083-5p GO:0030695

mmu-miR-3083-5p GO:0005343

mmu-miR-3083-5p GO:0016638

mmu-miR-3083-5p GO:0048037

mmu-miR-3083-5p GO:0060589

mmu-miR-3083-5p GO:0017134

mmu-miR-3083-5p GO:0015296

mmu-miR-3083-5p GO:0005547

mmu-miR-3083-5p GO:0050661

mmu-miR-3083-5p GO:0016709

mmu-miR-3083-5p GO:0015370

mmu-miR-3083-5p GO:0008047

mmu-miR-3083-5p GO:0004714

\section{Description}

flavin adenine dinucleotide binding

D-amino-acid oxidase activity

fatty acid synthase activity

voltage-gated chloride channel activity

anion transmembrane transporter activity

coenzyme binding

acidic amino acid transmembrane transporter activity

chloride ion binding

voltage-gated anion channel activity

GTPase activator activity

FAD binding

P-value P-value adjust Q-value Gene

$\begin{array}{lll}0.0018 & 0.001787878 & 0.0675 \mathrm{Dao} / F m o 2\end{array}$

$\begin{array}{lll}0.0086 & 0.008637936 & 0.0675 \text { Dao }\end{array}$

$\begin{array}{lll}0.0086 & 0.008637936 & 0.0675 \text { Elovl6 }\end{array}$

$\begin{array}{lll}0.0086 & 0.008637936 & 0.0675 \text { Clcnka }\end{array}$

$0.0091 \quad 0.009075254 \quad 0.0675$ Slc13a3/Clcnka

$\begin{array}{lll}0.0095 & 0.009457615 & 0.0675 \mathrm{Dao} / \mathrm{Fmo} 2\end{array}$

$\begin{array}{lll}0.0102 & 0.01020088 & 0.0675 \text { Slc13a3 }\end{array}$

$\begin{array}{lll}0.0102 & 0.01020088 & 0.0675 \text { Clcnka }\end{array}$

$\begin{array}{lll}0.0125 & 0.012540932 & 0.0675 \text { Clcnka }\end{array}$

$\begin{array}{lll}0.0137 & 0.013666935 & 0.0675 \text { Arhgef15/Adap1 }\end{array}$

oxidoreductase activity, acting on the $\mathrm{CH}-\mathrm{NH} 2$ group of donors, oxys 0.0149

GTPase regulator activity

organic acid:sodium symporter activity

oxidoreductase activity, acting on the $\mathrm{CH}-\mathrm{NH} 2$ group of donors

cofactor binding

nucleoside-triphosphatase regulator activity

fibroblast growth factor binding

anion:cation symporter activity

phosphatidylinositol-3,4,5-trisphosphate binding

NADP binding

0.016

0.014098063

0.0675 Dao

0.0172

0.016026054

0.0675 Dao

oxidoreductase activity, acting on paired donors, with incorporation

solute:sodium symporter activity

enzyme activator activity

transmembrane receptor protein tyrosine kinase activity $\begin{array}{lll}0.0185 & 0.018547865 & 0.0675 \text { Dao/Fmo2 }\end{array}$

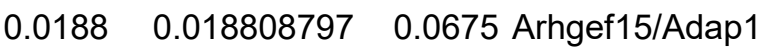

$\begin{array}{lll}0.0203 & 0.020303427 & 0.0689 \text { Fgfr } 1\end{array}$

$\begin{array}{lll}0.0226 & 0.022620914 & 0.0727 \text { Slc13a3 }\end{array}$

$\begin{array}{lll}0.0288 & 0.028775593 & 0.0878 \text { Adap } 1\end{array}$

$\begin{array}{lll}0.0303 & 0.030308533 & 0.0881 \mathrm{Fmo} 2\end{array}$

$\begin{array}{ll}0.03260366 & 0.0905 \mathrm{Fmo} 2\end{array}$

$\begin{array}{lll}0.0379 & 0.03793902 & 0.1007 \text { Slc13a3 }\end{array}$

$\begin{array}{lll}0.0422 & 0.042151259 & 0.1072 \\ 0.047 & 0.047020752 & 0.1148 \text { Fgfr15/Adap1 }\end{array}$

$0.047 \quad 0.047020752 \quad 0.1148$ Fgfr 1 
mmu-miR-466l-3p mmu-miR-466I-3p mmu-miR-466l-3p mmu-miR-466l-3p mmu-miR-466l-3p mmu-miR-466l-3p mmu-miR-466I-3p mmu-miR-466I-3p mmu-miR-466l-3p mmu-miR-466l-3p mmu-miR-466l-3p mmu-miR-466I-3p mmu-miR-466I-3p mmu-miR-466l-3p mmu-miR-466l-3p mmu-miR-466l-3p mmu-miR-466I-3p mmu-miR-466l-3p mmu-miR-466l-3p mmu-miR-466l-3p mmu-miR-466I-3p mmu-miR-466I-3p mmu-miR-466I-3p mmu-miR-466l-3p mmu-miR-466I-3p mmu-miR-466l-3p mmu-miR-466I-3p mmu-miR-466l-3p mmu-miR-466l-3p mmu-miR-466l-3p mmu-miR-466l-3p mmu-miR-466I-3p mmu-miR-466l-3p mmu-miR-466l-3p mmu-miR-466l-3p mmu-miR-466I-3p mmu-miR-466l-3p mmu-miR-466l-3p
GO:0016627 GO:0004935 GO:0001227 GO:0035240 GO:0016722 GO:0004694 GO:1901338 GO:0031690 GO:0008969 GO:0017161 GO:0019178 GO:0043726 GO:0043813 GO:0052825 GO:0052828 GO:0052829 GO:0052830 GO:0052831 GO:0052867 GO:1990003 GO:0003785 GO:0008579 GO:0030351 GO:0030352 GO:0030487 GO:0043812 GO:0052827 GO:0000252 GO:0004495 GO:0008875 GO:0018451 GO:0018452 GO:0018453 GO:0019152 GO:0032442 GO:0032866 GO:0032867 GO:0033709 oxidoreductase activity, acting on the $\mathrm{CH}-\mathrm{CH}$ group of donors adrenergic receptor activity

transcriptional repressor activity, RNA polymerase II transcription re dopamine binding

oxidoreductase activity, oxidizing metal ions

eukaryotic translation initiation factor 2alpha kinase activity

catecholamine binding

adrenergic receptor binding

phosphohistidine phosphatase activity

inositol-1,3,4-trisphosphate 4-phosphatase activity

NADP phosphatase activity

5-amino-6-(5-phosphoribitylamino)uracil phosphatase activity

phosphatidylinositol-3,5-bisphosphate 5-phosphatase activity inositol-1,3,4,5,6-pentakisphosphate 1-phosphatase activity

inositol-3,4-bisphosphate 4-phosphatase activity

inositol-1,3,4-trisphosphate 1-phosphatase activity

inositol-1,3,4,6-tetrakisphosphate 6-phosphatase activity

inositol-1,3,4,6-tetrakisphosphate 1-phosphatase activity

phosphatidylinositol-1,4,5-trisphosphate 5-phosphatase activity

IDP phosphatase activity

actin monomer binding

JUN kinase phosphatase activity

inositol-1,3,4,5,6-pentakisphosphate 3-phosphatase activity

inositol-1,4,5,6-tetrakisphosphate 6-phosphatase activity

inositol-4,5-bisphosphate 5-phosphatase activity

phosphatidylinositol-4-phosphate phosphatase activity

inositol pentakisphosphate phosphatase activity

C-3 sterol dehydrogenase (C-4 sterol decarboxylase) activity

mevaldate reductase activity

gluconate dehydrogenase activity

epoxide dehydrogenase activity

5-exo-hydroxycamphor dehydrogenase activity

2-hydroxytetrahydrofuran dehydrogenase activity acetoin dehydrogenase activity

phenylcoumaran benzylic ether reductase activity

$D$-xylose:NADP reductase activity

L-arabinose:NADP reductase activity

$\mathrm{D}$-arabinitol dehydrogenase, D-ribulose forming (NADP+) activity
$0.0031 \quad 0.00314403 \quad 0.0186$ Cybrd1/Kcnab2

$0.0074 \quad 0.007420631 \quad 0.0186$ Adrb1

$0.007856846 \quad 0.0186$ Arid5b/Atf3

$0.0089 \quad 0.008898524 \quad 0.0186$ Adrb1

$0.0104 \quad 0.010374346 \quad 0.0186$ Cybrd1

$0.0111 \quad 0.011111481 \quad 0.0186$ Eif2ak2

$\begin{array}{lll}0.0118 & 0.0118481 & 0.0186 \text { Adrb1 }\end{array}$

$0.0126 \quad 0.012584202 \quad 0.0186$ Adrb1

$\begin{array}{lll}0.017 & 0.016989987 & 0.0186 \text { Ptpdc1 }\end{array}$

$\begin{array}{lll}0.017 & 0.016989987 & 0.0186 \text { Ptpdc1 }\end{array}$

$0.017 \quad 0.016989987 \quad 0.0186$ Ptpdc1

$\begin{array}{lll}0.017 & 0.016989987 & 0.0186 \text { Ptpdc1 }\end{array}$

$0.017 \quad 0.016989987 \quad 0.0186$ Ptpdc1

$\begin{array}{lll}0.017 & 0.016989987 & 0.0186 \text { Ptpdc1 }\end{array}$

\begin{tabular}{lll}
0.017 & 0.016989987 & 0.0186 \\
\hline
\end{tabular}

$\begin{array}{lll}0.017 & 0.016989987 & 0.0186 \text { Ptpdc1 }\end{array}$

$\begin{array}{lll}0.017 & 0.016989987 & 0.0186 \text { Ptpdc1 }\end{array}$

$\begin{array}{lll}0.017 & 0.016989987 & 0.0186 \text { Ptpdc1 }\end{array}$

$\begin{array}{lll}0.017 & 0.016989987 & 0.0186 \text { Ptpdc1 }\end{array}$

$\begin{array}{lll}0.017 & 0.016989987 & 0.0186 \text { Ptpdc1 }\end{array}$

$0.0177 \quad 0.017722483 \quad 0.0186$ Pknox2

$0.01770 .017722483 \quad 0.0186$ Ptpdc1

$0.0177 \quad 0.017722483 \quad 0.0186$ Ptpdc1

$\begin{array}{lll}0.0177 & 0.017722483 & 0.0186 \text { Ptpdc1 }\end{array}$

$0.0177 \quad 0.017722483 \quad 0.0186$ Ptpdc1

$0.0177 \quad 0.017722483 \quad 0.0186$ Ptpdc1

$0.0177 \quad 0.017722483 \quad 0.0186$ Ptpdc1

$0.0185 \quad 0.018454466 \quad 0.0186$ Kcnab2

$0.0185 \quad 0.018454466 \quad 0.0186$ Kcnab2

$0.0185 \quad 0.018454466 \quad 0.0186$ Kcnab2

$0.01850 .018454466 \quad 0.0186$ Kcnab2

$0.0185 \quad 0.018454466 \quad 0.0186$ Kcnab2

$0.0185 \quad 0.018454466 \quad 0.0186$ Kcnab2

$0.0185 \quad 0.018454466 \quad 0.0186$ Kcnab2

$0.0185 \quad 0.018454466 \quad 0.0186$ Kcnab2

$0.0185 \quad 0.018454466 \quad 0.0186$ Kcnab2

$0.0185 \quad 0.018454466 \quad 0.0186$ Kcnab2

$0.0185 \quad 0.018454466 \quad 0.0186$ Kcnab2 
mmu-miR-466l-3p mmu-miR-466I-3p mmu-miR-466l-3p mmu-miR-466l-3p mmu-miR-466l-3p mmu-miR-466l-3p mmu-miR-466I-3p mmu-miR-466I-3p mmu-miR-466l-3p mmu-miR-466l-3p mmu-miR-466l-3p mmu-miR-466I-3p mmu-miR-466I-3p mmu-miR-466I-3p mmu-miR-466l-3p mmu-miR-466l-3p mmu-miR-466I-3p mmu-miR-466l-3p mmu-miR-466I-3p mmu-miR-466l-3p mmu-miR-466I-3p mmu-miR-466I-3p mmu-miR-466I-3p mmu-miR-466l-3p mmu-miR-466I-3p mmu-miR-466l-3p mmu-miR-466I-3p mmu-miR-466I-3p mmu-miR-466I-3p mmu-miR-466l-3p mmu-miR-466l-3p mmu-miR-466I-3p mmu-miR-466l-3p mmu-miR-466l-3p mmu-miR-466l-3p mmu-miR-466I-3p mmu-miR-466l-3p mmu-miR-466I-3p
GO:0034831 GO:0034840 GO:0035380 GO:0043713 GO:0044103 GO:0044105 GO:0048258 GO:0052677 GO:0008330 GO:0019198 GO:0035410 GO:0098519 GO:0016312 GO:0051990 GO:0052629 GO:0052743 GO:0034594 GO:0033765 GO:0034596 GO:0052744 GO:0004448 GO:0034595 GO:0046030 GO:0000978 GO:0008748 GO:0017017 GO:0043738 GO:0043826 GO:0043883 GO:0052693 GO:0004032 GO:0033549 GO:0043914 GO:0015459 GO:0034593 GO:0000987 GO:0001159 GO:0000982
(R)-(-)-1,2,3,4-tetrahydronaphthol dehydrogenase activity

3-hydroxymenthone dehydrogenase activity

very long-chain-3-hydroxyacyl-CoA dehydrogenase activity (R)-2-hydroxyisocaproate dehydrogenase activity

L-arabinose 1-dehydrogenase (NADP+) activity

L-xylulose reductase $(N A D+)$ activity

3-ketoglucose-reductase activity

D-arabinitol dehydrogenase, D-xylulose forming (NADP+) activity

protein tyrosine/threonine phosphatase activity

transmembrane receptor protein phosphatase activity

dihydrotestosterone 17-beta-dehydrogenase activity

nucleotide phosphatase activity, acting on free nucleotides

inositol bisphosphate phosphatase activity

(R)-2-hydroxyglutarate dehydrogenase activity

phosphatidylinositol-3,5-bisphosphate 3-phosphatase activity

inositol tetrakisphosphate phosphatase activity

phosphatidylinositol trisphosphate phosphatase activity

steroid dehydrogenase activity, acting on the $\mathrm{CH}-\mathrm{CH}$ group of dono

phosphatidylinositol phosphate 4-phosphatase activity

phosphatidylinositol monophosphate phosphatase activity

isocitrate dehydrogenase activity

phosphatidylinositol phosphate 5-phosphatase activity

inositol trisphosphate phosphatase activity

RNA polymerase II core promoter proximal region sequence-specifi

$\mathrm{N}$-ethylmaleimide reductase activity

MAP kinase tyrosine/serine/threonine phosphatase activity

reduced coenzyme F420 dehydrogenase activity

sulfur oxygenase reductase activity

malolactic enzyme activity

epoxyqueuosine reductase activity

alditol:NADP+1-oxidoreductase activity

MAP kinase phosphatase activity

NADPH:sulfur oxidoreductase activity

potassium channel regulator activity

phosphatidylinositol bisphosphate phosphatase activity

core promoter proximal region sequence-specific DNA binding

core promoter proximal region DNA binding

transcription factor activity, RNA polymerase II core promoter proxin $\begin{array}{lll}0.0185 & 0.018454466 & 0.0186 \mathrm{Kcnab2}\end{array}$

$\begin{array}{lll}0.0185 & 0.018454466 & 0.0186 \text { Kcnab2 }\end{array}$

$\begin{array}{lll}0.0185 & 0.018454466 & 0.0186 \text { Kcnab2 }\end{array}$

$\begin{array}{lll}0.0185 & 0.018454466 & 0.0186 \mathrm{Kcnab} 2\end{array}$

$\begin{array}{lll}0.0185 & 0.018454466 & 0.0186 \text { Kcnab2 }\end{array}$

$\begin{array}{lll}0.0185 & 0.018454466 & 0.0186 \mathrm{Kcnab} 2\end{array}$

$\begin{array}{lll}0.0185 & 0.018454466 & 0.0186 \mathrm{Kcnab2}\end{array}$

$\begin{array}{lll}0.0185 & 0.018454466 & 0.0186 \text { Kcnab2 }\end{array}$

$\begin{array}{lll}0.0192 & 0.019185935 & 0.0186 \text { Ptpdc1 }\end{array}$

$\begin{array}{lll}0.0192 & 0.019185935 & 0.0186 \text { Ptpdc1 }\end{array}$

$0.0192 \quad 0.019185935 \quad 0.0186$ Kcnab2

$\begin{array}{lll}0.0192 & 0.019185935 & 0.0186 \text { Ptpdc1 }\end{array}$

$\begin{array}{lll}0.0199 & 0.019916891 & 0.0186 \text { Ptpdc1 }\end{array}$

$\begin{array}{lll}0.0199 & 0.019916891 & 0.0186 \text { Kcnab2 }\end{array}$

$\begin{array}{lll}0.0199 & 0.019916891 & 0.0186 \text { Ptpdc1 }\end{array}$

$\begin{array}{lll}0.0199 & 0.019916891 & 0.0186 \text { Ptpdc1 }\end{array}$

$\begin{array}{lll}0.0206 & 0.020647334 & 0.0188 \text { Ptpdc1 }\end{array}$

$\begin{array}{lll}0.0214 & 0.021377265 & 0.0188 \text { Kcnab2 }\end{array}$

$\begin{array}{lll}0.0214 & 0.021377265 & 0.0188 \text { Ptpdc1 }\end{array}$

$\begin{array}{lll}0.0221 & 0.022106684 & 0.0188 \text { Ptpdc1 }\end{array}$

$\begin{array}{lll}0.0228 & 0.022835591 & 0.0188 \text { Kcnab2 }\end{array}$

$\begin{array}{lll}0.0228 & 0.022835591 & 0.0188 \text { Ptpdc1 }\end{array}$

$\begin{array}{lll}0.0236 & 0.023563987 & 0.0188 \text { Ptpdc1 }\end{array}$

$0.0255 \quad 0.025513422 \quad 0.0188$ Atf3/Zic1

$\begin{array}{llll}0.0257 & 0.02574611 & 0.0188 \text { Cybrd1 }\end{array}$

$\begin{array}{llll}0.0257 & 0.02574611 & 0.0188 \text { Ptpdc1 }\end{array}$

$\begin{array}{llll}0.0257 & 0.02574611 & 0.0188 \text { Cybrd1 }\end{array}$

$\begin{array}{llll}0.0257 & 0.02574611 & 0.0188 \text { Cybrd1 }\end{array}$

$\begin{array}{lll}0.0257 & 0.02574611 & 0.0188 \text { Cybrd1 }\end{array}$

$\begin{array}{llll}0.0257 & 0.02574611 & 0.0188 \text { Cybrd1 }\end{array}$

$\begin{array}{lll}0.0265 & 0.026472463 & 0.0188 \text { Kcnab2 }\end{array}$

$\begin{array}{llll}0.0265 & 0.026472463 & 0.0188 \text { Ptpdc1 }\end{array}$

$\begin{array}{lll}0.0265 & 0.026472463 & 0.0188 \text { Cybrd1 }\end{array}$

$\begin{array}{lll}0.0279 & 0.027923642 & 0.0193 \text { Kcnab2 }\end{array}$

$\begin{array}{lll}0.0279 & 0.027923642 & 0.0193 \text { Ptpdc1 }\end{array}$

$\begin{array}{llll}0.0284 & 0.028388631 & 0.0194 & \text { Atf3/Zic1 }\end{array}$

$\begin{array}{lll}0.0288 & 0.028830844 & 0.0194 \text { Atf3/Zic1 }\end{array}$

$\begin{array}{lll}0.0293 & 0.02927587 & 0.0195 \text { Atf3/Zic1 }\end{array}$


mmu-miR-466l-3p mmu-miR-466I-3p mmu-miR-466l-3p mmu-miR-466l-3p mmu-miR-466l-3p mmu-miR-466l-3p mmu-miR-466I-3p mmu-miR-466I-3p mmu-miR-466l-3p mmu-miR-466I-3p mmu-miR-466l-3p mmu-miR-466I-3p mmu-miR-466l-3p mmu-miR-883a-5p mmu-miR-883a-5p mmu-miR-883a-5p mmu-miR-883a-5p mmu-miR-883a-5p mmu-miR-883a-5p mmu-miR-883a-5p mmu-miR-883a-5p mmu-miR-883a-5p mmu-miR-883a-5p mmu-miR-883a-5p mmu-miR-883a-5p mmu-miR-883a-5p mmu-miR-883a-5p mmu-miR-883a-5p mmu-miR-883a-5p mmu-miR-883a-5p mmu-miR-883a-5p mmu-miR-883a-5p mmu-miR-15b-5p mmu-miR-15b-5p mmu-miR-15b-5p mmu-miR-15b-5p mmu-miR-15b-5p mmu-miR-15b-5p
GO:0008106 GO:0052745 GO:0042605 GO:0052866 GO:0004033 GO:0033764 GO:0004715 GO:0016229 GO:0003712 GO:0008138 GO:0016628 GO:0000989 GO:0000988 GO:0004713 GO:0004028 GO:0017127 GO:0004694 GO:0015248 GO:0004029 GO:0005272 GO:0001786 GO:0022804 GO:0022839 GO:1901618 GO:0016620 GO:0004715 GO:0000049 GO:0016903 GO:0004714 GO:0005518 GO:0072341 GO:0003725 GO:0050660 GO:0050662 GO:0034713 GO:0048185 GO:0070411 GO:0030676 alcohol dehydrogenase (NADP+) activity

inositol phosphate phosphatase activity

peptide antigen binding

phosphatidylinositol phosphate phosphatase activity

aldo-keto reductase (NADP) activity

steroid dehydrogenase activity, acting on the $\mathrm{CH}-\mathrm{OH}$ group of donor

non-membrane spanning protein tyrosine kinase activity

steroid dehydrogenase activity

transcription cofactor activity

protein tyrosine/serine/threonine phosphatase activity

oxidoreductase activity, acting on the $\mathrm{CH}-\mathrm{CH}$ group of donors, NAD

transcription factor activity, transcription factor binding

transcription factor activity, protein binding

protein tyrosine kinase activity

3-chloroallyl aldehyde dehydrogenase activity

cholesterol transporter activity

eukaryotic translation initiation factor 2alpha kinase activity

sterol transporter activity

aldehyde dehydrogenase (NAD) activity

sodium channel activity

phosphatidylserine binding

active transmembrane transporter activity

ion gated channel activity

organic hydroxy compound transmembrane transporter activity

oxidoreductase activity, acting on the aldehyde or oxo group of donc

non-membrane spanning protein tyrosine kinase activity

tRNA binding

oxidoreductase activity, acting on the aldehyde or oxo group of donc

transmembrane receptor protein tyrosine kinase activity

collagen binding

modified amino acid binding

double-stranded RNA binding

flavin adenine dinucleotide binding

coenzyme binding

type I transforming growth factor beta receptor binding

activin binding

I-SMAD binding

Rac guanyl-nucleotide exchange factor activity
0.0301

0.0301

0.0308

0.0308

0.0323

$0.030096593 \quad 0.0195$ Kcnab2 $0.030096593 \quad 0.0195$ Ptpdc1

$0.030819893 \quad 0.0195 \mathrm{H} 2-\mathrm{T} 24$

$0.030819893 \quad 0.0195$ Ptpdc1

0.0322649710 .0199 Kcnab2

$\begin{array}{ll}0.032264971 & 0.0199 \text { Kcnab2 }\end{array}$

$\begin{array}{lll}0.033 & 0.03298675 & 0.0201 \text { Eif2ak2 }\end{array}$

$\begin{array}{llll}0.0359 & 0.0358688 & 0.0216 \text { Kcnab2 }\end{array}$

$\begin{array}{lll}0.0371 & 0.037095292 & 0.0221 \text { Arid5b/Aip }\end{array}$

$\begin{array}{lll}0.043 & 0.043038597 & 0.0253 \text { Ptpdc1 }\end{array}$

$\begin{array}{lll}0.0452 & 0.045179731 & 0.0262 \text { Cybrd1 }\end{array}$

$\begin{array}{lll}0.0469 & 0.046882995 & 0.0269 \\ \text { Arid5b/Aip }\end{array}$

$\begin{array}{lll}0.0478 & 0.047783333 & 0.0271 \text { Arid5b/Aip }\end{array}$

$0.0037 \quad 0.003717585 \quad 0.1079$ Eif2ak2/Ret

$0.007 \quad 0.006985499 \quad 0.1079$ Aldh8a1

$\begin{array}{lll}0.0091 & 0.009072211 & 0.1079 \text { Abcg4 }\end{array}$

$\begin{array}{lll}0.0105 & 0.010461068 & 0.1079 \\ 0.0 i f 2 a k 2\end{array}$

$\begin{array}{lll}0.0105 & 0.010461068 & 0.1079 \mathrm{Abcg} 4\end{array}$

$\begin{array}{lll}0.0146 & 0.014616697 & 0.122 \text { Aldh8a1 }\end{array}$

$\begin{array}{lll}0.0236 & 0.023564498 & 0.122 \text { Asic1 }\end{array}$

$0.0249 \quad 0.024934303 \quad 0.122$ Cd300a

$0.0262 \quad 0.026235228 \quad 0.122$ Abcg4/Slc38a4

$\begin{array}{lll}0.0277 & 0.027668509 & 0.122 \text { Asic1 }\end{array}$

$\begin{array}{lll}0.029 & 0.029032916 & 0.122 \text { Abcg } 4\end{array}$

$\begin{array}{lll}0.0297 & 0.029714445 & 0.122 \text { Aldh8a1 }\end{array}$

$\begin{array}{lll}0.0311 & 0.03107616 & 0.122 \text { Eif2ak2 }\end{array}$

$\begin{array}{lll}0.0331 & 0.033115373 & 0.122 \text { Ptcd1 }\end{array}$

$0.0378579 \quad 0.1271$ Aldh8a1

$\begin{array}{lll}0.0419 & 0.041905557 & 0.1271 \text { Ret }\end{array}$

$\begin{array}{lll}0.0419 & 0.041905557 & 0.1271 \text { Itga11 }\end{array}$

$\begin{array}{lll}0.0486 & 0.048616196 & 0.1295 \mathrm{Cd} 300 \mathrm{a}\end{array}$

\begin{tabular}{lll}
0.0493 & 0.049284831 & 0.1295 \\
\hline
\end{tabular}

$0.0009 \quad 0.000921882 \quad 0.0301$ Aifm2/Fmo2

$\begin{array}{lll}0.005 & 0.004953449 & 0.0401 \text { Aifm2/Fmo2 }\end{array}$

$\begin{array}{lll}0.0057 & 0.005679073 & 0.0401 \mathrm{Smad} 7\end{array}$

$\begin{array}{llll}0.0062 & 0.00624534 & 0.0401 \text { Smad7 }\end{array}$

$\begin{array}{llll}0.0062 & 0.00624534 & 0.0401 \mathrm{Smad} 7\end{array}$

$0.00790 .007942354 \quad 0.0401$ Dock2 
mmu-miR-15b-5p mmu-miR-15b-5p mmu-miR-15b-5p mmu-miR-15b-5p mmu-miR-15b-5p mmu-miR-15b-5p mmu-miR-15b-5p mmu-miR-15b-5p mmu-miR-15b-5p mmu-miR-15b-5p mmu-miR-15b-5p mmu-miR-15b-5p mmu-miR-15b-5p mmu-miR-15b-5p mmu-miR-15b-5p mmu-miR-15b-5p mmu-miR-15b-5p mmu-miR-15b-5p mmu-miR-15b-5p mmu-miR-15b-5p mmu-miR-15b-5p mmu-miR-15b-5p mmu-miR-15b-5p mmu-miR-188-3p mmu-miR-188-3p mmu-miR-188-3p mmu-miR-188-3p mmu-miR-188-3p mmu-miR-188-3p mmu-miR-188-3p mmu-miR-188-3p mmu-miR-188-3p mmu-miR-188-3p mmu-miR-188-3p mmu-miR-188-3p mmu-miR-188-3p mmu-miR-188-3p mmu-miR-188-3p
GO:0004806 GO:0048037 GO:0016645 GO:0001540 GO:0008748 GO:0043738 GO:0043826 GO:0043883 GO:0052693 GO:0043914 GO:0050661 GO:0016709 GO:0005160 GO:0005546 GO:0005518 GO:1902936 GO:0016628 GO:0019843 GO:0005089 GO:0046332 GO:0008013 GO:0019905 GO:0008172 GO:0043121 GO:0017127 GO:0015248 GO:0017075 GO:0008373 GO:0001786 GO:1901618 GO:0004714 GO:0005518 GO:0048306 GO:0072341 GO:0019199 GO:0019905 GO:0005319 GO:0042626 triglyceride lipase activity

cofactor binding

oxidoreductase activity, acting on the $\mathrm{CH}-\mathrm{NH}$ group of donors

beta-amyloid binding

$\mathrm{N}$-ethylmaleimide reductase activity

reduced coenzyme $\mathrm{F} 420$ dehydrogenase activity

sulfur oxygenase reductase activity

malolactic enzyme activity

epoxyqueuosine reductase activity

NADPH:sulfur oxidoreductase activity

NADP binding

oxidoreductase activity, acting on paired donors, with incorporation

transforming growth factor beta receptor binding

phosphatidylinositol-4,5-bisphosphate binding

collagen binding

phosphatidylinositol bisphosphate binding

oxidoreductase activity, acting on the $\mathrm{CH}-\mathrm{CH}$ group of donors, NAD

rRNA binding

Rho guanyl-nucleotide exchange factor activity

SMAD binding

beta-catenin binding

syntaxin binding

S-methyltransferase activity

neurotrophin binding

cholesterol transporter activity

sterol transporter activity

syntaxin-1 binding

sialyltransferase activity

phosphatidylserine binding

organic hydroxy compound transmembrane transporter activity

transmembrane receptor protein tyrosine kinase activity

collagen binding

calcium-dependent protein binding

modified amino acid binding

transmembrane receptor protein kinase activity

syntaxin binding

lipid transporter activity

ATPase activity, coupled to transmembrane movement of substanc $\begin{array}{lll}0.0091 & 0.009072211 & 0.0401 \text { Lipe }\end{array}$

$\begin{array}{lll}0.0098 & 0.009826868 & 0.0401 \mathrm{Aifm} 2 / \mathrm{Fmo} 2\end{array}$

$\begin{array}{lll}0.0153 & 0.015265219 & 0.0414 \text { Aifm2 }\end{array}$

$\begin{array}{lll}0.0175 & 0.01750835 & 0.0414 \text { Apba1 }\end{array}$

$\begin{array}{lll}0.0197 & 0.019746763 & 0.0414 \text { Aifm2 }\end{array}$

$\begin{array}{lll}0.0197 & 0.019746763 & 0.0414 \text { Aifm2 }\end{array}$

$\begin{array}{lll}0.0197 & 0.019746763 & 0.0414 \text { Aifm2 }\end{array}$

$\begin{array}{lll}0.0197 & 0.019746763 & 0.0414 \text { Aifm2 }\end{array}$

$\begin{array}{lll}0.0197 & 0.019746763 & 0.0414 \text { Aifm2 }\end{array}$

$\begin{array}{lll}0.0203 & 0.02030563 & 0.0414 \text { Aifm2 }\end{array}$

$\begin{array}{lll}0.022 & 0.021980468 & 0.0422 \mathrm{Fmo} 2\end{array}$

\begin{tabular}{lll}
0.0237 & 0.023652662 & 0.0429 \\
\hline
\end{tabular}

$\begin{array}{lll}0.0292 & 0.029207602 & 0.0493 \mathrm{Smad} 7\end{array}$

$\begin{array}{lll}0.0331 & 0.033078685 & 0.0493 \text { Apba1 }\end{array}$

$\begin{array}{lll}0.0342 & 0.034182089 & 0.0493 \text { Smad7 }\end{array}$

$\begin{array}{lll}0.0342 & 0.034182089 & 0.0493 \text { Apba1 }\end{array}$

$\begin{array}{lll}0.0347 & 0.034733355 & 0.0493 \text { Aifm2 }\end{array}$

$\begin{array}{lll}0.0375 & 0.037485331 & 0.0504 \text { Lipe }\end{array}$

$\begin{array}{lll}0.0386 & 0.038584092 & 0.0504 \text { Dock2 }\end{array}$

$\begin{array}{llll}0.0424 & 0.042420644 & 0.0532 \text { Smad7 }\end{array}$

$\begin{array}{llll}0.0468 & 0.046787968 & 0.0551 & \text { Smad7 }\end{array}$

$\begin{array}{lll}0.0484 & 0.048420968 & 0.0551 \mathrm{Napa}\end{array}$

$\begin{array}{lll}0.049 & 0.048964728 \quad 0.0551 \mathrm{Bhmt}\end{array}$

$0.0048 \quad 0.004807263 \quad 0.0231$ Hap1

$\begin{array}{lll}0.0057 & 0.005679073 & 0.0231 \text { Abcg } 4\end{array}$

$\begin{array}{llll}0.0066 & 0.006550195 & 0.0231 \text { Abcg4 }\end{array}$

$\begin{array}{llll}0.0087 & 0.008724997 & 0.0231 & \text { Cplx2 }\end{array}$

$\begin{array}{llll}0.0092 & 0.009159443 & 0.0231 & \text { St3gal1 }\end{array}$

$\begin{array}{lll}0.0157 & 0.015655599 & 0.0314 \mathrm{Cd} 300 \mathrm{a}\end{array}$

$\begin{array}{lll}0.0182 & 0.01824331 & 0.0314 \text { Abcg4 }\end{array}$

$\begin{array}{lll}0.0264 & 0.026397389 & 0.0314 \text { Ret }\end{array}$

$\begin{array}{lll}0.0264 & 0.026397389 & 0.0314 \text { Itga11 }\end{array}$

$\begin{array}{lll}0.029 & 0.028959668 & 0.0314 \mathrm{Cplx} 2\end{array}$

$\begin{array}{llll}0.0307 & 0.030664481 & 0.0314 \mathrm{Cd} 300 \mathrm{a}\end{array}$

$\begin{array}{lll}0.037 & 0.037033565 & 0.0314 \text { Ret }\end{array}$

$\begin{array}{lll}0.0375 & 0.037456828 & 0.0314 \text { Cplx2 }\end{array}$

$\begin{array}{lll}0.0438 & 0.043785718 & 0.0314 \text { Abcg } 4\end{array}$

$\begin{array}{lll}0.0471 & 0.047145789 & 0.0314 \text { Abcg4 }\end{array}$ 
mmu-miR-188-3p mmu-miR-188-3p mmu-miR-188-3p mmu-miR-326-5p mmu-miR-326-5p mmu-miR-326-5p mmu-miR-326-5p mmu-miR-326-5p mmu-miR-326-5p mmu-miR-326-5p mmu-miR-326-5p mmu-miR-326-5p mmu-miR-326-5p mmu-miR-326-5p mmu-miR-326-5p mmu-miR-326-5p mmu-miR-326-5p mmu-miR-326-5p mmu-miR-326-5p mmu-miR-326-5p

\section{KEGG pathways}

\section{Cluster}

mmu-miR-3083-5p mmu00511 mmu-miR-3083-5p mmu00512 mmu-miR-3083-5p mmu01040 mmu-miR-3083-5p mmu00062 mmu-miR-3083-5p mmu00260 mmu-miR-3083-5p mmu00330 mmu-miR-3083-5p mmu01212 mmu-miR-466l-3p mmu05168 mmu-miR-466l-3p mmu05167 mmu-miR-466l-3p mmu05169 mmu-miR-466l-3p mmu05203 mmu-miR-466l-3p mmu05166 mmu-miR-466l-3p mmu05165 mmu-miR-466I-3p hydrolase activity, acting on acid anhydrides, catalyzing transmembr P-P-bond-hydrolysis-driven transmembrane transporter activity primary active transmembrane transporter activity cholesterol transporter activity

sterol transporter activity

syntaxin-1 binding

sialyltransferase activity

sodium channel activity

phosphatidylserine binding

ion gated channel activity

organic hydroxy compound transmembrane transporter activity

transmembrane receptor protein tyrosine kinase activity

collagen binding

calcium-dependent protein binding

structural constituent of cytoskeleton

modified amino acid binding

transmembrane receptor protein kinase activity

syntaxin binding

protein tyrosine phosphatase activity

lipid transporter activity

$\begin{array}{rrl}0.0476 & 0.04756505 & 0.0314 \text { Abcg4 } \\ 0.0492 & 0.049240434 & 0.0314 \text { Abcg4 } \\ 0.0497 & 0.049658865 & 0.0314 \text { Abcg4 } \\ 0.0062 & 0.00624534 & 0.0837 \text { Abcg4 } \\ 0.0072 & 0.007203008 & 0.0837 \text { Abcg4 } \\ 0.0096 & 0.009593508 & 0.0837 \text { Cplx2 } \\ 0.0101 & 0.01007098 & 0.0837 \text { St3gal1 } \\ 0.0163 & 0.016259098 & 0.0837 \text { Asic1 } \\ 0.0172 & 0.017207989 & 0.0837 \text { Cd300a } \\ 0.0191 & 0.019103274 & 0.0837 \text { Asic1 } \\ 0.02 & 0.02004967 & 0.0837 \text { Abcg4 } \\ 0.029 & 0.028999122 & 0.0837 \text { Ret } \\ 0.029 & 0.028999122 & 0.0837 \text { Itga11 } \\ 0.0318 & 0.031809789 & 0.0837 \text { Cplx2 } \\ 0.0327 & 0.032745033 & 0.0837 \text { Tubb3 } \\ 0.0337 & 0.033679456 & 0.0837 \text { Cd300a } \\ 0.0407 & 0.040661511 & 0.0837 \text { Ret } \\ 0.0411 & 0.041125346 & 0.0837 \text { Cplx2 } \\ 0.0444 & 0.044366491 & 0.0837 \text { Ptpn7 } \\ 0.0481 & 0.048058459 & 0.0837 \text { Abcg4 }\end{array}$

Description

Other glycan degradation

Mucin type O-glycan biosynthesis

Biosynthesis of unsaturated fatty acids

Fatty acid elongation

Glycine, serine and threonine metabolism

Arginine and proline metabolism

Fatty acid metabolism

Herpes simplex infection

Kaposi sarcoma-associated herpesvirus infection

Epstein-Barr virus infection

Viral carcinogenesis

Human T-cell leukemia virus 1 infection

Human papillomavirus infection

Mineral absorption
P-value P-value adjust Q-value Gene

$\begin{array}{lll}0.0151 & 0.015133 & 0.0989 \mathrm{Hexdc}\end{array}$

$\begin{array}{lll}0.0235 & 0.023455 \quad 0.0989 \text { Gcnt1 }\end{array}$

$\begin{array}{lll}0.0235 & 0.023455 & 0.0989 \text { Elovl6 }\end{array}$

$\begin{array}{lll}0.0268 & 0.026767 & 0.0989 \text { Elovl6 }\end{array}$

$\begin{array}{lll}0.0334 & 0.033362 & 0.0989 \text { Dao }\end{array}$

$\begin{array}{lll}0.0416 & 0.041552 & 0.0989 \text { Dao }\end{array}$

$\begin{array}{lll}0.0432 & 0.043183 & 0.0989 \text { Elovl6 }\end{array}$

$0.0129 \quad 0.012946 \quad 0.1094$ Eif2ak2/H2-T24

$0.0132 \quad 0.013178 \quad 0.1094$ Eif2ak2/H2-T24

$0.0147 \quad 0.01473 \quad 0.1094$ Eif2ak2/H2-T24

$0.0149 \quad 0.014853 \quad 0.1094$ Eif2ak2/H2-T24

$0.0217 \quad 0.021698 \quad 0.122$ Atf3/H2-T24

$\begin{array}{lll}0.0361 & 0.036066 & 0.122 \text { Eif2ak2/H2-T24 }\end{array}$

$\begin{array}{lll}0.0375 & 0.037464 & 0.122 \text { Cybrd1 }\end{array}$ 
mmu-miR-466l-3p mmu-miR-883a-5p mmu-miR-883a-5p mmu-miR-883a-5p mmu-miR-883a-5p mmu-miR-883a-5p mmu-miR-15b-5p mmu-miR-15b-5p mmu-miR-188-3p mmu-miR-188-3p mmu-miR-188-3p mmu-miR-188-3p mmu-miR-188-3p mmu-miR-188-3p mmu-miR-326-5p mmu-miR-326-5p mmu-miR-326-5p mmu-miR-326-5p mmu-miR-326-5p mmu-miR-326-5p mmu04923

mmu04392

mmu05340

mmu05216

mmu05165

mmu02010

mmu00512

mmu00260

mmu00533

mmu00604

mmu00603

mmu00512

mmu05216

mmu02010

mmu00533

$\mathrm{mmu00604}$

mmu00603

mmu00512

mmu05216

mmu02010
Regulation of lipolysis in adipocytes

Hippo signaling pathway - multiple species

Primary immunodeficiency

Thyroid cancer

Human papillomavirus infection

ABC transporters

Mucin type O-glycan biosynthesis

Glycine, serine and threonine metabolism

Glycosaminoglycan biosynthesis - keratan sulfate

Glycosphingolipid biosynthesis - ganglio series

Glycosphingolipid biosynthesis - globo and isoglobo series

Mucin type O-glycan biosynthesis

Thyroid cancer

ABC transporters

Glycosaminoglycan biosynthesis - keratan sulfate

Glycosphingolipid biosynthesis - ganglio series

Glycosphingolipid biosynthesis - globo and isoglobo series

Mucin type O-glycan biosynthesis

Thyroid cancer

ABC transporters
0.0456

0.0218

$0.045624 \quad 0.122$ Adrb1

$0.021796 \quad 0.1535$ Tead4

$0.0300690 .1535 \mathrm{Cd} 8 \mathrm{a}$

$0.030893 \quad 0.1535$ Ret

0.0309

0.0361

0.0391

0.0301

0.0427

0.0118

0.0126

0.0135

0.0235

0.0309

0.0391

0.0135

0.0144

0.0154

0.0268

0.0352

0.0446
$0.036066 \quad 0.1535$ Eif2ak2/ltga11

$0.039101 \quad 0.1535$ Abcg4

$0.030059 \quad 0.172$ Gcnt1

$0.042693 \quad 0.172 \mathrm{Bhmt}$

$0.011787 \quad 0.0614$ St3gal1

$0.012625 \quad 0.0614$ St3gal1

$0.0134610 .0614 \mathrm{St3gal} 1$

$0.023455 \quad 0.0768$ St3gal1

$0.030893 \quad 0.0768$ Ret

$0.039101 \quad 0.0768$ Abcg4

$0.013461 \quad 0.0755$ St3gal1

$0.014416 \quad 0.0755$ St3gal1

$0.01537 \quad 0.0755$ St3gal1

$0.026762 \quad 0.094$ St3gal1

$0.03523 \quad 0.094$ Ret

$0.044563 \quad 0.094$ Abcg 4 